Research Article

\title{
Optimal Surrender Policy of Guaranteed Minimum Maturity Benefits in Variable Annuities with Regime-Switching Volatility
}

\author{
Xiankang Luo $\mathbb{D}$ and Jie Xing $\mathbb{D}$ \\ Faculty of Science, Yibin University, Yibin 644000, China \\ Correspondence should be addressed to Jie Xing; xingjie8531@163.com
}

Received 28 March 2021; Accepted 23 June 2021; Published 14 July 2021

Academic Editor: Stefania Tomasiello

Copyright () 2021 Xiankang Luo and Jie Xing. This is an open access article distributed under the Creative Commons Attribution License, which permits unrestricted use, distribution, and reproduction in any medium, provided the original work is properly cited.

\begin{abstract}
This study investigates valuation of guaranteed minimum maturity benefits (GMMB) in variable annuity contract in the case where the guarantees can be surrendered at any time prior to the maturity. In the event of the option being exercised early, early surrender charges will be applied. We model the underlying mutual fund dynamics under regime-switching volatility. The valuation problem can be reduced to an American option pricing problem, which is essentially an optimal stopping problem. Then, we obtain the pricing partial differential equation by a standard Markovian argument. A detailed discussion shows that the solution of the problem involves an optimal surrender boundary. The properties of the optimal surrender boundary are given. The regime-switching Volterra-type integral equation of the optimal surrender boundary is derived by probabilistic methods. Furthermore, a sensitivity analysis is performed for the optimal surrender decision. In the end, we adopt the trinomial tree method to determine the optimal strategy.
\end{abstract}

\section{Introduction}

A variable annuity (VA) contract is issued by an insurance company, which offers upside opportunities in financial market and also protects against downside risk by adding various types of benefit riders. In a VA contract, the policyholder agrees to pay either a single payment or a series of periodic premiums to his/her account. These payments are typically invested in risky financial assets in the market for better benefits. Due to the uncertainty of the market, the VA contract provides various benefits in the event of poor performance of financial risky assets. These guarantees embedded in VA contracts are similar to financial options. For instance, a guaranteed minimum accumulation benefit (GMAB) guarantees a lump sum amount at a predetermined future date, a guaranteed minimum death benefit (GMDB) guarantees a minimum lump sum upon death, a guaranteed minimum living benefit (GMLB) provides income guarantees to protect the policyholder's income from declining, and a guaranteed minimum maturity benefit (GMMB) is a guarantee that provides the policyholder with a minimum benefit on maturity of the VA contract (see the works of Bernard et al. [1], Ignatieva et al. [2], Bauer et al. [3], and Shen et al. [4]).

The following is a brief literature review. Bacinello [5] computed the value of participating life insurance policies with surrender options using a recursive binomial tree approach. Bernard et al. [1] split the value of VA contract into a European part and an early exercise premium and derived a Volterra-type integral equation with respect to the optimal surrender boundary under the assumption that the underlying risky asset follows the geometric Brownian motion (GBM) model. Alonso-Garcia et al. [6] considered VA contract with GMAB rider when policyholder's proceeds are taxed. Shen et al. [4] considered a VA contract with a GMMB rider and proposed a numerical approach to the value function of the VA contract and the optimal surrender boundary. Kang and Ziveyi [7] analyzed how the policyholder surrender behavior is influenced by changes in various sources of risk impacting a VA contract under stochastic volatility and interest rate. Jeon and Kwak [8] studied VA contract with GMMB riders for path-dependent 
guarantees and applied Mellin transform to calculate the value function and the optimal surrender strategy. Jeon and Kim [9] derived an analytic pricing formula for a VA contract with GMMB rider by using the Laplace-Carson transform. MacKay and Ocejo [10] studied a portfolio optimization problem involving a VA contract with GMMB rider and solved constrained and unconstrained version of the optimization problem.

The majority of the literature has been premised under the GBM framework. However, it is well known that the stochastic variability in the market parameters is not reflected in the GBM model. In filling this gap, we should extend the results presented in Shen et al. [4] to the stochastic volatility model. The GBM model has been extended in various ways. Among those generalizations, the regimeswitching model has become a popular model. In this paper, we focus on a VA contract embedded with a GMMB rider by incorporating regime-switching volatility. This problem can be viewed as an optimal stopping problem with regimeswitching. It is interesting to point out that our problem has a lot in common with the well-known American option in financial mathematics. However, the gain function in our problem is time dependent, which is different from the American option pricing problem. There are some papers that have dealt with the optimal stopping problem with regime-switching volatility. Buffington and Elliott [11] discussed American options in a regime-switching market and obtained an approximate valuation of American option. Jacka and Ocejo [12] studied the regularity of the value function for a finite-horizon optimal stopping problem in the presence of regime-switching uncertainty. Boyarchenkp and Levendorski [13] calculated the early exercise boundaries for American options by using a generalization of Carr's randomization procedure for regime-switching models. In the insurance literature, Siu [14] considered the fair valuation of a participating life insurance policy with surrender options for regime-switching models and provided a decomposition result for the value of the policy. In a word, the above literature mainly concentrated on the value functions and none of them discussed the properties of the free boundary rigorously. Yang [15] studied the properties of free boundary arising from Russian option pricing problem with regime-switching volatility by the variational inequality approach.

The main contributions of this paper are stated as follows. Firstly, we model the underlying mutual fund dynamics under regime-switching volatility. The related variational inequality is given by a standard Markovian argument. Secondly, compared with the method used in [15], we derive some basic properties of the corresponding value function and the optimal surrender boundary by probabilistic methods. Besides, to derive the regimeswitching Volterra-type integral equations of the optimal surrender boundary, we use dynamic programming principle to convert the regime-switching optimal stopping problem into an optimal stopping problem with constantcoefficient geometric Brownian motion, which makes the proof significant in methodology. Furthermore, since there has been less focus on the development of a realistic modelling framework for analyzing the impact of various sources of risk in influencing the surrender behavior, it is crucial to make rigorous sensitivity analysis when we make risk management decision. Compared with the sensitivity analysis in Shen et al. [4], which was performed by numerical examples without proofs, we apply a rigorous variational inequality approach to explore the impacts of varying model parameters on the optimal surrender boundary. Finally, we calculate the optimal surrender boundary by trinomial tree method (see [16]) and perform numerical examples to analyze the policyholder's surrender decisions.

The remaining parts of this paper are organized as follows. In Section 2, we state the valuation problem of a VA contract with GMMB rider under regime-switching volatility. In Section 3, we state the main results in this paper. In Section 4, we give some numerical experiments to verify our theoretical results. In Section 5, we give detailed proofs of the results in Section 3. In Section 6, we conclude the study.

\section{Formulation of the Problem}

We consider a probability space $(\Omega, \mathscr{F}, \mathbb{Q})$, where $\mathbb{Q}$ is a risk neutral measure. Also, we consider a standard Brownian motion $W=\left\{W_{t}, t \geq 0\right\}$. Assume that the continuous time Markov chain $\alpha=\left\{\alpha_{t}, t \geq 0\right\}$ takes values in $\delta=\{1,2\}$ and the generator of $\alpha_{t}$ is of the form

$$
A=\left(\begin{array}{cc}
-\lambda_{1} & \lambda_{1} \\
\lambda_{2} & -\lambda_{2}
\end{array}\right) \text {, }
$$

where $\lambda_{1}$ and $\lambda_{2}$ are positive constants. Let $\mathbb{F}=\left\{\mathscr{F}_{t}, 0 \leq t \leq T\right\}$ be the natural filtration, which is generated by the standard Brownian motion $W$ and Markov chain $\alpha$ and satisfies the usual condition. Let $\mathbb{E}[\cdot]$ denote the expectation under risk neutral measure $\mathbb{Q}$. We consider the behavior of the policyholder who is involved in a VA contract embedded with a GMMB rider, where the contract can be surrendered anytime prior to maturity subject to penalty charges. The policyholder pays the premium at the contract initialization and then invests the money in a fund consisting of units of an underlying asset $S=\left\{S_{t}, 0 \leq t \leq T\right\}$, whose risk neutral dynamic evolves under the influence of regime-switching volatility, which is specified as follows:

$$
\mathrm{d} S_{t}=r S_{t} \mathrm{~d} t+\sigma\left(\alpha_{t}\right) S_{t} \mathrm{~d} W_{t},
$$

where $r>0$ is the risk-free interest rate and $\sigma\left(\alpha_{t}\right) \in\left\{\sigma_{1}, \sigma_{2}\right\}$ is the volatility of the underlying asset. The resulting mutual fund's value is given by

$$
F_{t}=e^{-c t} S_{t}
$$

where $c$ is the continuously compounded insurance charge expressed in percentage terms by the annuity provider (see [4]). A direct computation shows that the dynamic of the fund value $F=\left\{F_{t}, 0 \leq t \leq T\right\}$ satisfies

$$
\mathrm{d} F_{t}=(r-c) F_{t} \mathrm{~d} t+\sigma\left(\alpha_{t}\right) F_{t} \mathrm{~d} W_{t} .
$$

We assume that the guaranteed amount $G_{t} \equiv G>0$ is a constant throughout the life of the contract. Denote that 
$\max (a, 0):=a^{+}$. Since the insurer will provide $\max \left(G, F_{T}\right)$ to the policyholder at maturity date, hence, under risk neutral argument, the initial value of the VA contract is given by

$$
\mathbb{E}\left[e^{-r T} \max \left(G, F_{T}\right)\right]=\mathbb{E}\left[e^{-r T} F_{T}\right]+\mathbb{E}\left[e^{-r T}\left(G-F_{T}\right)^{+}\right],
$$

where $F_{T}$ is the fund value at terminal time $T$, with $G$ being the guaranteed value at maturity of the contract. In order to find the fair insurance charge $c^{*}$ during the time $t \in[0, T]$, we should set the initial fund value $F_{0}$ to be equal to the value in (5). In (5), we decompose the value of the VA contract into two components, which was presented in [4] firstly.

In view of (5), we may find it optimal to surrender the contract early for avoiding higher fees which are proportional to the fund value. In the event of the guarantee being exercised prior to the maturity, we assume that the surrender benefit equals to

$$
\left(1-\kappa_{t}\right) F_{t}
$$

where $\kappa_{t}$ is a penalty percentage charged for surrendering at time $t \in[0, T]$. As in Bernard et al. [1] and Shen et al. [4], $\kappa_{t}$ is exponentially decreasing with time and is equal to

$$
1-e^{-\kappa(T-t)} \text {. }
$$

Then, the surrender benefit becomes

$$
e^{-\kappa(T-t)} F_{t}
$$

In this paper, we assume that $\kappa<c<r$ holds. Otherwise, it is optimal to surrender the option at maturity $T$.

We notice that the policyholder will receive account value $F_{T}$ from the mutual fund and the benefit $\left(G-F_{T}\right)^{+}$ from the embedded guarantee without a surrender option. More precisely, the value of the VA contract at time $t$ in this case is given by

$$
\begin{aligned}
\mathbb{E}\left[e^{-r(T-t)} \max \left(F_{T}, G\right) \mid \mathscr{F}_{t}\right]= & \mathbb{E}\left[e^{-r(T-t)} F_{T} \mid \mathscr{F}_{t}\right] \\
& +\mathbb{E}\left[e^{-r(T-t)}\left(G-F_{T}\right)^{+} \mid \mathscr{F}_{t}\right] .
\end{aligned}
$$

On the contrary, if there is a surrender option for the embedded guarantee, then the policyholder is allowed to surrender the guarantee at any time $t$ before the maturity and the surrender benefit will be

$$
\left(G-e^{-\kappa(T-t)} F_{t}\right)^{+}
$$

Besides, the policyholder will also receive his/her account value $F_{T}$ at maturity. Then, the value of the VA contract at time $t$ can be written as follows:

$$
\begin{aligned}
\Phi(t, f, i):= & e^{-r(T-t)} \mathbb{E}\left[F_{T} \mid \mathscr{F}_{t}\right] \\
& +\sup _{t \leq \tau \leq T} \mathbb{E}\left[e^{-r(\tau-t)}\left(G-e^{-\kappa(T-\tau)} F_{\tau}\right)^{+} \mid \mathscr{F}_{t}\right] .
\end{aligned}
$$

By the formula in [17] and put-call parity, we can derive a formula for European call option under regime-switching volatility. Then, by letting $E \longrightarrow 0$, we can obtain an analytical formula for the first component in the above equation, where $E$ is the strike price of the call option. Now, we focus on the second term and denote

$$
P(t, f, i):=\sup _{t \leq \tau \leq T} \mathbb{E}\left[e^{-r(\tau-t)}\left(G-e^{-\kappa(T-\tau)} F_{\tau}\right)^{+} \mid \mathscr{F}_{t}\right] .
$$

We set $Y_{t}=e^{-\kappa(T-t)} F_{t}, \quad y=e^{-\kappa(T-t)} f, \quad$ and $P\left(t, e^{\kappa(T-t)} y, i\right)=V(t, y, i)$, where the dynamic of $Y$ satisfies

$$
\mathrm{d} Y_{t}=(r-c+\kappa) Y_{t} \mathrm{~d} t+\sigma\left(\alpha_{t}\right) Y_{t} \mathrm{~d} W_{t} .
$$

Solving (13) with initial condition $Y_{0}=y$, we find that $Y_{t}:=y M_{t}=y \exp \left(\int_{0}^{t}\left(r-c+\kappa-\frac{1}{2} \sigma\left(\alpha_{s}\right)^{2}\right) \mathrm{d} s+\int_{0}^{t} \sigma\left(\alpha_{s}\right) \mathrm{d} W_{s}\right)$.

Then, by Markovian property of $Y$, we derive that

$$
\begin{aligned}
V(t, y, i) & =\sup _{t \leq \tau \leq T} \mathbb{E}\left[e^{-r(\tau-t)} h\left(Y_{\tau}\right) \mid \alpha_{t}=i, Y_{t}=y\right] \\
& =\sup _{0 \leq \tau \leq T-t} \mathbb{E}^{i, y}\left[e^{-r \tau} h\left(Y_{\tau}\right)\right],
\end{aligned}
$$

where $\mathbb{E}^{i, y}[\cdot]$ means $\mathbb{E}\left[\cdot \mid \alpha_{0}=i, Y_{0}=y\right]$ and $h(y)=(G-y)^{+}$.

\section{Main Results}

Having stated main problem in Section 2, we formulate the main assertions of this paper, which are built on probabilistic methods of optimal stopping problem developed by Peskir and Shiryaev [18] and variational inequality approach by Yang [15]. Now, we show some useful properties of the value function defined in (15), which will be frequently used later. Firstly, we introduce the infinitesimal generator of $\left(Y_{t}, \alpha_{t}\right)$ as follows:

$$
\begin{aligned}
(\mathscr{A} f)(t, y, i):= & (\mathscr{L} f)(t, y, i) \\
& +\lambda_{i}(f(t, y, j)-f(t, y, i)), \quad j \neq i,
\end{aligned}
$$

where

$$
(\mathscr{L} f)(t, y, i):=\frac{1}{2} \sigma_{i}^{2} y^{2} f_{y y}(t, y, i)+(r-c+\kappa) y f_{y}(t, y, i),
$$

where $f_{y}$ denotes $(\partial f(t, y, i) / \partial y)$ and $f_{y y}$ is defined similarly.

Lemma 1. The value function $V$ satisfies the following variational inequality:

$$
\begin{array}{r}
\min \left\{\left(r V-V_{t}-\mathscr{A} V\right)(t, y, i), V(t, y, i)-h(y)\right\}=0, \\
(t, y, i) \in(0, T) \times(0,+\infty) \times \mathcal{S},
\end{array}
$$

with terminal condition,

$$
V(T, y, i)=h(y) \text {. }
$$


Proof. The proof is easy, and we refer to Appendix A.

Lemma 2. The value function $V(t, y, i)$ is positive, nonincreasing in $t$ and $y$, and convex in $y$.

Proof. The proof is easy, and we refer to Appendix B.

Lemma 3. $V(t, \cdot, i)$ is Lipschitz continuous in $\mathbb{R}^{+}$and $V(\cdot, y, i)$ is 1/2-Hölder continuous uniformly over a neighborhood of $y$.

Proof. See Section 5.

To discuss the optimal exercise boundary, we introduce the stopping region and continuation region as follows:

$$
\begin{aligned}
\mathscr{D} & :=\{(t, y, i) \in[0, T] \times(0,+\infty) \times \mathcal{S}: V(t, y, i)=h(y)\}, \\
\mathscr{C} & :=\{(t, y, i) \in[0, T] \times(0,+\infty) \times \mathcal{S}: V(t, y, i)>h(y)\}, \\
\mathscr{D}_{i} & :=\{(t, y) \in[0, T] \times(0,+\infty): V(t, y, i)=h(y)\}, \quad i \in \mathcal{S}, \\
\mathscr{C}_{i} & :=\{(t, y) \in[0, T] \times(0,+\infty): V(t, y, i)>h(y)\}, \quad i \in \mathcal{S} .
\end{aligned}
$$

From [18], we know that the optimal stopping time for problem (15) can be represented as

$$
\tau_{\mathscr{D}}=\inf \left\{0 \leq s \leq T-t:\left(t+s, Y_{s}^{y}, \alpha_{s}\right) \in \mathscr{D}\right\} \wedge(T-t) .
$$

To investigate the shape of the stopping region, we need the following important lemma.

Lemma 4. The function $y \mapsto V(t, y, i)+y$ is increasing. Moreover, $\mathscr{D}$ is downward connected: if $\left(t, y^{\prime}, i\right) \in \mathscr{D}$, then $(t, y, i) \in \mathscr{D}$, for $y<y^{\prime}$.

Proof. See Section 5.

Now, we are prepared to describe the shape of the stopping region. The result is as follows.
Theorem 1. There exists a continuous function $b_{i}(t)$ such that

$$
b_{i}(t)=\sup \{y>0: V(t, y, i)=h(y)\}, \quad i \in \mathcal{S} .
$$

Moreover, $b_{i}(\cdot)$ is nondecreasing and

$$
b_{i}(T)=\min \left\{G, \frac{r G}{c-\kappa}\right\} .
$$

Proof. See Section 5.

By the earlier assumption that $r>c>\kappa$, it turns out that $b_{i}(T)=G$ for $i \in \mathcal{S}$. The next theorem shows that we can transform the original optimal stopping problem into a free boundary problem.

Theorem 2. The value function $V$ defined in (15) is the unique solution to the following free boundary problem:

$$
\begin{gathered}
V_{t}(t, y, i)+(\mathscr{A} V)(t, y, i)-r V(t, y, i) \\
=0 \text { in } \mathscr{C}=\left\{(t, y, i): y>b_{i}(t)\right\},
\end{gathered}
$$

$$
V\left(t, b_{i}(t), i\right)=h\left(b_{i}(t)\right), \quad t \in[0, T), i \in \mathcal{S},
$$

$V_{y}\left(t, b_{i}(t), i\right)=-1, \quad t \in[0, T), i \in \mathcal{S}$

$$
V(T, y, i)=h(y), \quad y \in(0,+\infty), i \in \mathcal{S} .
$$

In particular, the partial derivatives $V_{y y}, V_{y}$, and $V_{t}$ exist and are continuous in $\mathscr{C}$.

Proof. See Section 5.

Next, we introduce the following notations to obtain the regime-switching Volterra-type integral equations:

$$
\begin{gathered}
J_{i}(t, y):=\mathbb{E}\left[e^{-\left(r+\lambda_{i}\right)(T-t)} h\left(Y_{T-t}^{(i)}\right)\right], \\
K_{i}\left(t, y, u, b_{i}(u+t)\right):=\mathbb{E}\left[e^{-\left(r+\lambda_{i}\right) u}\left(\Phi_{i}\left(Y_{u}^{(i)}\right) \chi_{\left\{Y_{u}^{(i)}<b_{i}(u+t)\right\}}-\lambda_{i} V\left(t+u, Y_{u}^{(i)}, j\right) \chi_{\left\{Y_{u}^{(i)}>b_{i}(u+t)\right\}}\right)\right], \quad j \neq i,
\end{gathered}
$$


where

$$
\Phi_{i}(y)=\left(c-r+\lambda_{i}\right) y-\left(r+\lambda_{i}\right) G
$$

$Y_{t}^{(i)}$ satisfies

$$
\mathrm{d} Y_{t}^{(i)}=Y_{t}^{(i)}\left((r-c+\kappa) \mathrm{d} t+\sigma_{i} \mathrm{~d} W_{t}\right)
$$

with initial condition $Y_{0}^{(i)}=y$.

Theorem 3. The value function defined in (15) is given by $V(t, y, i)=J_{i}(t, y)-\int_{0}^{T-t} K_{i}\left(t, y, u, b_{i}(u+t)\right) \mathrm{d} u, \quad i \in \mathcal{S}$.

Furthermore, the free boundary $b_{i}(t)$ satisfies the following nonlinear regime-switching Volterra integral equations:

$$
\begin{aligned}
h\left(b_{i}(t)\right)= & J_{i}\left(t, b_{i}(t)\right) \\
& -\int_{0}^{T-t} K_{i}\left(t, b_{i}(t), u, b_{i}(u+t)\right) \mathrm{d} u, \quad i \in \mathcal{S} .
\end{aligned}
$$

Proof. See Section 5.

Remark 1. In this remark, we shall calculate $J_{i}$ and $K_{i}$ in Theorem 3. By (31), we have $Y_{t}^{(i)}=y \exp \{(r-c+\kappa-(1 /$ 2) $\left.\left.\sigma_{i}^{2}\right) t+\sigma_{i} W_{t}\right\}$. We denote $\tau:=T-t$ and $Z:=-\left(W_{\tau} / \sqrt{\tau}\right)$. Then, $Z$ follows a standard normal distribution. We set $d_{-}(\tau, y ; G):=\left(\left(r-c+\kappa-(1 / 2) \sigma_{i}^{2}\right) \tau+\log (y / G)\right) / \sigma_{i} \sqrt{\tau}$ and $d_{+}(\tau, y ; G):=d_{-}(\tau, y ; G)+\sigma_{i} \sqrt{\tau}$. By a direct computation,

$$
\begin{aligned}
J_{i}(t, y) & =\mathbb{E}\left[e^{-\left(r+\lambda_{i}\right) \tau}\left(G-y \exp \left(-\sigma_{i} \sqrt{\tau} Z+\left(r-c+\kappa-\frac{1}{2} \sigma_{i}^{2}\right) \tau\right)\right)^{+}\right] \\
& =\frac{1}{\sqrt{2 \pi}} \int_{-\infty}^{+\infty} e^{-\left(r+\lambda_{i}\right) \tau}\left(G-y \exp \left(-\sigma_{i} \sqrt{\tau} z+\left(r-c+\kappa-\frac{1}{2} \sigma_{i}^{2}\right) \tau\right)\right)^{+} e^{-\left(z^{2} / 2\right)} \mathrm{d} z \\
& =\frac{1}{\sqrt{2 \pi}} \int_{d_{-}(\tau, y ; G)}^{+\infty} e^{-\left(r+\lambda_{i}\right) \tau}\left(G-y \exp \left(-\sigma_{i} \sqrt{\tau} z+\left(r-c+\kappa-\frac{1}{2} \sigma_{i}^{2}\right) \tau\right)\right) e^{-\left(z^{2} / 2\right)} \mathrm{d} z \\
& =G e^{-\left(r+\lambda_{i}\right) \tau} \frac{1}{\sqrt{2 \pi}} \int_{d_{-}(\tau, y ; G)}^{+\infty} e^{-\left(z^{2} / 2\right)} \mathrm{d} z-y e^{-\left(c-\kappa+\lambda_{i}\right) \tau} \frac{1}{\sqrt{2 \pi}} \int_{d_{-}(\tau, y ; G)}^{+\infty} e^{-(1 / 2)\left(z+\sigma_{i} \sqrt{\tau}\right)^{2}} \mathrm{~d} z \\
& =G e^{-\left(r+\lambda_{i}\right) \tau} N\left(-d_{-}(\tau, y ; G)\right)-y e^{-\left(c-\kappa+\lambda_{i}\right) \tau} N\left(-d_{+}(\tau, y ; G)\right),
\end{aligned}
$$

where $N(y)=(1 / \sqrt{2 \pi}) \int_{-\infty}^{y} e^{-\left(z^{2} / 2\right)} \mathrm{d} z$.

To calculate $K_{i}$, we denote $\widetilde{y}_{i}(z, u):=y \exp$ $\left(-\sigma_{i} \sqrt{u} z+\left(r-c+\kappa-(1 / 2) \sigma_{i}^{2}\right) u\right)$. Then, we obtain that

$$
\begin{aligned}
& -\lambda_{i} \mathbb{E}\left[e^{-\left(r+\lambda_{i}\right) u} V\left(t+u, Y_{u}^{(i)}, j\right) X_{\left\{Y_{u}^{(i)}>b_{i}(u+t)\right\}}\right] \\
& =-\lambda_{i} e^{-\left(r+\lambda_{i}\right) u} \mathbb{E}\left[V\left(t+u, Y_{u}^{(i)}, j\right) X_{\left\{Y_{u}^{(i)}>b_{i}(u+t)\right\}}\right] \\
& =-\frac{\lambda_{i}}{\sqrt{2 \pi}} \int_{-\infty}^{+\infty} V\left(t+u, \widetilde{y}_{i}(z, u), j\right) X_{\left\{\tilde{y}_{i}(z, u)>b_{i}(u+t)\right\}} e^{-\left(z^{2} / 2\right)} \mathrm{d} z \\
& =-\frac{\lambda_{i}}{\sqrt{2 \pi}} \int_{-\infty}^{d_{-}\left(u, y ; b_{i}(u+t)\right)} V\left(t+u, \tilde{y}_{i}(z, u), j\right) e^{-\left(z^{2} / 2\right)} \mathrm{d} z,
\end{aligned}
$$

and by definition of $\Phi_{i}$ (see (30)), 


$$
\begin{aligned}
\mathbb{E} & {\left[e^{-\left(r+\lambda_{i}\right) u} \Phi_{i}\left(Y_{u}^{(i)}\right) X_{\left\{Y_{u}^{(i)}<b_{i}(u+t)\right\}}\right] } \\
= & \frac{e^{-\left(r+\lambda_{i}\right) u}}{\sqrt{2 \pi}} \int_{-\infty}^{+\infty} \Phi_{i}\left(\tilde{y}_{i}(z, u)\right) X_{\left\{\tilde{y}_{i}(z, u)<b_{i}(u+t)\right\}} e^{-\left(z^{2} / 2\right)} \mathrm{d} z \\
= & \frac{e^{-\left(r+\lambda_{i}\right) u}}{\sqrt{2 \pi}} \int_{d_{-}\left(u, y ; b_{i}(u+t)\right)}^{+\infty} \Phi_{i}\left(\tilde{y}_{i}(z, u)\right) e^{-\left(z^{2} / 2\right)} \mathrm{d} z \\
= & \frac{\left(c-\kappa+\lambda_{i}\right) y e^{-\left(r+\lambda_{i}\right) u}}{\sqrt{2 \pi}} \int_{d_{-}\left(u, y ; b_{i}(u+t)\right)}^{+\infty} e^{-\left(z^{2} / 2\right)-\sigma_{i} \sqrt{u} z+\left(r-c+\kappa-(1 / 2) \sigma_{i}^{2}\right) u} \mathrm{~d} z \\
& -\left(r+\lambda_{i}\right) G N\left(-d_{-}\left(u, y ; b_{i}(u+t)\right)\right) \\
= & \frac{\left(c-\kappa+\lambda_{i}\right) y e^{\left(-c+\kappa-\lambda_{i}\right) u}}{\sqrt{2 \pi}} \int_{d_{+}\left(u, y ; b_{i}(u+t)\right)}^{+\infty} e^{-\left(z^{2} / 2\right)} \mathrm{d} z-\left(r+\lambda_{i}\right) G N\left(-d_{-}\left(u, y ; b_{i}(u+t)\right)\right) \\
= & \left(c-\kappa+\lambda_{i}\right) y e^{-\left(c-\kappa+\lambda_{i}\right) u} N\left(-d_{+}\left(u, y ; b_{i}(u+t)\right)\right)-\left(r+\lambda_{i}\right) G N\left(-d_{-}\left(u, y ; b_{i}(u+t)\right)\right) .
\end{aligned}
$$

Hence,

$$
\begin{aligned}
K_{i}\left(t, y, u, b_{i}(u+t)\right)= & \left(c-\kappa+\lambda_{i}\right) y e^{-\left(c-\kappa+\lambda_{i}\right) u} N\left(-d_{+}\left(u, y ; b_{i}(u+t)\right)\right) \\
& -\left(r+\lambda_{i}\right) G N\left(-d_{-}\left(u, y ; b_{i}(u+t)\right)\right) \\
& -\frac{\lambda_{i}}{\sqrt{2 \pi}} \int_{-\infty}^{d_{-}\left(u, y ; b_{i}(u+t)\right)} V\left(t+u, \widetilde{y}_{i}(z, u), j\right) e^{-\left(z^{2} / 2\right)} \mathrm{d} z .
\end{aligned}
$$

At the end of this section, we present a sensitivity analysis for the impacts of model parameters on surrender strategies based on the variational inequality given in Lemma 1.

Theorem 4. Suppose $\sigma_{1}>\sigma_{2}$. Let $Y_{t}^{(i)}$ be defined as (31). Then,

$$
b_{1}^{0}(t) \leq b_{1}(t) \leq b_{2}(t) \leq b_{2}^{0}(t), \quad 0<t<T,
$$

where $b_{i}^{0}(i \in \mathcal{S})$ is the free boundary of the following optimal stopping problem:

$$
V_{i}^{0}(t, y)=\sup _{0 \leq \tau \leq T-t} \mathbb{E}^{y}\left[e^{-r \tau} h\left(Y_{\tau}^{(i)}\right)\right], \quad i \in \mathcal{S} .
$$

Proof. See Section 5.

Theorem 5. The free boundary $\left(b_{1}(t), b_{2}(t)\right)$ of problem (15) is decreasing with respect to $\sigma_{i}(i \in \mathcal{S})$ and $c$ and increasing with respect to $G, r$, and $\kappa$.

Proof. See Section 5.

\section{Numerical Examples}

In this section, we shall give some numerical examples to verify our theoretical findings. We now outline numerical techniques which will be used for calculating the optimal surrender boundary. The numerical method that we adopt was developed by Yuen and Yang [16] who implemented the trinomial tree method to solve the regime-switching option pricing problem. We will briefly explain how to use the trinomial tree method to solve our problem.

Let $\Delta t=T / n$ be the time step size. We take the jump ratios of the lattice as

$$
\begin{aligned}
& u=e^{\sigma \sqrt{\Delta t}}, \\
& d=e^{-\sigma \sqrt{\Delta t}},
\end{aligned}
$$

where

$$
\sigma=\max _{i \in \mathcal{S}}\left\{\sigma_{i}\right\}+(\sqrt{1.5}-1) \widetilde{\sigma}
$$

where $\widetilde{\sigma}$ is the arithmetic mean or geometric mean of $\sigma_{i}$, $i \in \mathcal{S}$. For regime $i$, let $\pi_{u}^{i}$, $\pi_{m}^{i}$, and $\pi_{d}^{i}$ be the risk neutral probabilities corresponding to the discounted fund value increases, remains the same, and decreases, respectively. By 
moment matching technique, the values of the probabilities are given by

$$
\begin{aligned}
& \pi_{m}^{i}=1-\frac{\sigma_{i}^{2}}{\sigma^{2}}, \\
& \pi_{u}^{i}=\frac{e^{(r-c+\kappa) \Delta t}-e^{-\sigma \sqrt{\Delta t}}-\left(1-\sigma^{2} / \sigma_{i}^{2}\right)\left(1-e^{-\sigma \sqrt{\Delta t}}\right)}{e^{\sigma \sqrt{\Delta t}}-e^{-\sigma \sqrt{\Delta t}}}, \\
& \pi_{d}^{i}=\frac{e^{\sigma \sqrt{\Delta t}}-e^{(r-c+\kappa) \Delta t}-\left(1-\sigma^{2} / \sigma_{i}^{2}\right)\left(e^{\sigma \sqrt{\Delta t}}-1\right)}{e^{\sigma \sqrt{\Delta t}}-e^{-\sigma \sqrt{\Delta t}}} .
\end{aligned}
$$

Let $y_{j+1}=u y_{j}$ and $y_{j-1}=\mathrm{d} y_{j}$, and denote by $V^{k}\left(y_{j}, i\right)$ the trinomial approximation of the primal value function for regime $i$ at asset price $y_{j}$ and time $t_{k}=k \Delta t$. Then, the trinomial formula of the primal value function is given by, for $k=0,1, \ldots, n-1$,

$$
V^{k}\left(y_{j}, i\right)=\max \left\{e^{-r \Delta t} \sum_{l=1}^{2} p_{i l}\left(\pi_{u}^{i} V^{k+1}\left(y_{j+1}, l\right)+\pi_{m}^{i} V^{k+1}\left(y_{j}, l\right)+\pi_{d}^{i} V^{k+1}\left(y_{j-1}, l\right)\right), h\left(y_{j}\right)\right\},
$$

with $V^{n}\left(y_{j}, i\right)=h\left(y_{j}\right)$ for $j=0, \pm 1, \ldots, \pm n$, in which $p_{i l}$ is the transition probability from state $i$ to state $l$ for the time interval with length $\Delta t$. It is given by

$$
\left(p_{i l}\right)_{i, l \in \mathcal{S}}=e^{A \Delta t}=I+\sum_{l=1}^{\infty}(\Delta t)^{l} \frac{A^{l}}{l !},
$$

where $I$ is the identity matrix and $A$ is the generator matrix of the Markov chain process given by (1).

Let $b^{k}(i) \approx b_{i}\left(t_{k}\right)$. The optimal surrender boundary can be calculated by

$$
\begin{aligned}
b^{k}(i) & =\max \left\{y_{j}: V^{k}\left(y_{j}, i\right)=h\left(y_{j}\right)\right\}, \\
k & =0,1, \ldots, n-1, i \in \mathcal{S} .
\end{aligned}
$$

Example 1. In this example, we assume the generator of the Markov chain $\alpha_{t}$ is taken as

$$
A=\left(\begin{array}{cc}
-0.5 & 0.5 \\
0.5 & -0.5
\end{array}\right),
$$

the terminal time $T=1$, and the time step of the trinomial tree model is equal to 10,000 .

(1) In Figure 1, we choose the penalty rate $\kappa=0.01$, the risk-free interest rate $r=0.06$, the insurance charge $c=0.02$, the volatility $\left(\sigma_{1}, \sigma_{2}\right)=(0.1,0.15)$ for Figure $1(\mathrm{a})$ and $\left(\sigma_{1}, \sigma_{2}\right)=(0.3,0.25)$ for Figure $1(\mathrm{~b})$, the minimum guarantee amount $G=100$. From Figure 1, we know that, at terminal time $T$, the surrender boundary value is equal to the minimum guarantee. The graph of the surrender boundary is generally increasing. Furthermore, Figure 1 shows how the optimal surrender boundary is influenced by the economic state. When the economy is in the state that the volatility is lower, the optimal surrender boundary is higher.
(2) In Figure 2, we assume that $G=100, c=0.02$, $\left(\sigma_{1}, \sigma_{2}\right)=(0.25,0.35)$, and $\kappa=0.01$. We depict the optimal surrender boundaries for $r=0.03,0.04$, $0.05,0.06$, respectively. This figure investigates how the interest rate affects the surrender decisions. It shows that increasing interest rate results in higher surrender boundary.

(3) In Figure 3, the parameters of our problem are given by $\quad r=0.06, \quad c=0.02, \quad \kappa=0.01, \quad$ and $\left(\sigma_{1}, \sigma_{2}\right)=(0.25,0.35)$. We plot the surrender boundaries in Figure 3. The minimum guarantee values are $G=75,100,125,150$, respectively. We note from this figure that the surrender boundaries are shifted upwards as the minimum guarantee value increases.

(4) In Figure 4, we assume that $G=100, r=0.06$, $\left(\sigma_{1}, \sigma_{2}\right)=(0.25,0.35)$, and $\kappa=0.01$. We analyze the sensitivity of the surrender boundaries with respect to the fee $c$. For $c=0.02,0.03,0.04,0.05$, we compute the surrender boundaries in this figure. It shows that a higher fee results in a lower surrender boundaries.

(5) In Figure 5, we take $G=100, r=0.06$, $\left(\sigma_{1}, \sigma_{2}\right)=(0.25,0.35)$, and $c=0.04$. This figure shows how the varied $\kappa$ impacts on the surrender boundaries. We vary the penalty rate $\kappa$ between 0 and 0.03 and compute the surrender boundaries. From this figure, we see that the surrender boundaries move up when $\kappa$ increases and the surrender incentive becomes much lower. Intuitively, increasing levels of $\kappa$ make it more expensive to surrender the guarantee early. Hence, it is rational to delay exercising the guarantee.

(6) In Figure 6, the parameters of the problem are given by $G=100, r=0.06, \kappa=0.01$, and $c=0.02$. This figure presents the impact of the volatility on the surrender boundaries. We choose the volatility as $\left(\sigma_{1}, \sigma_{2}\right)=(0.1,0.15), \quad(0.15,0.2), \quad(0.2,0.25)$, 


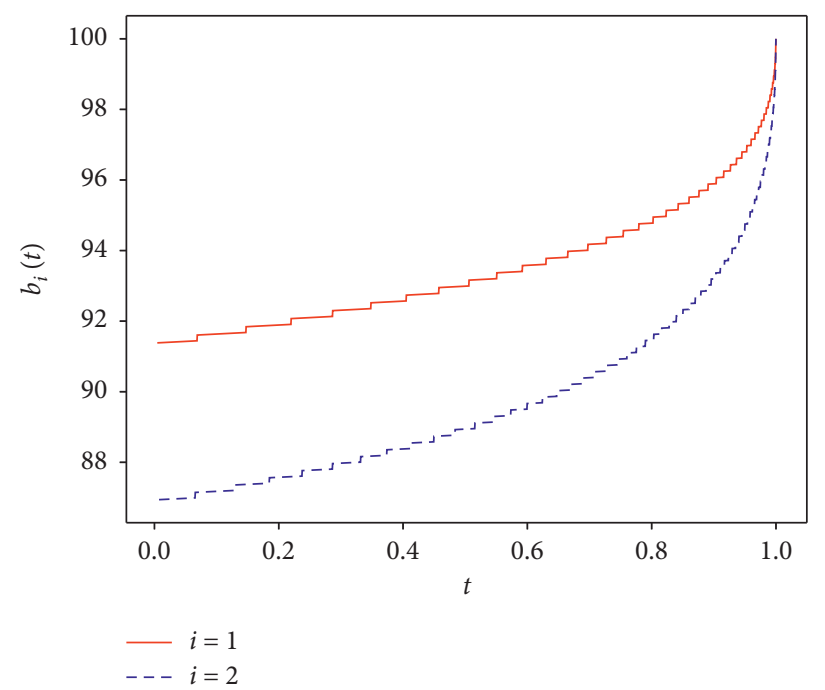

(a)

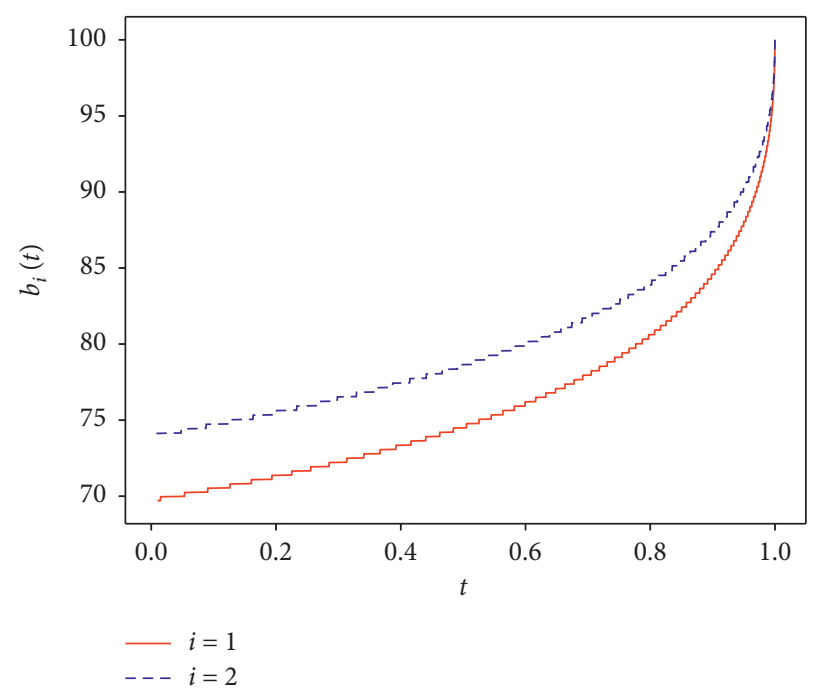

(b)

Figure 1: (a) Optimal surrender boundary for $\left(\sigma_{1}, \sigma_{2}\right)=(0.1,0.15)$; (b) optimal surrender boundary for $\left(\sigma_{1}, \sigma_{2}\right)=(0.3,0.25)$.

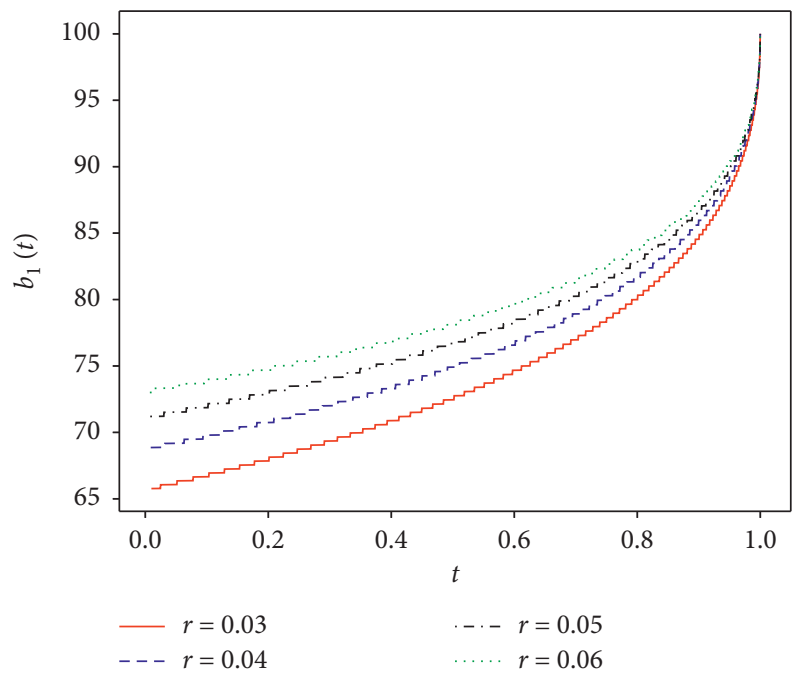

(a)

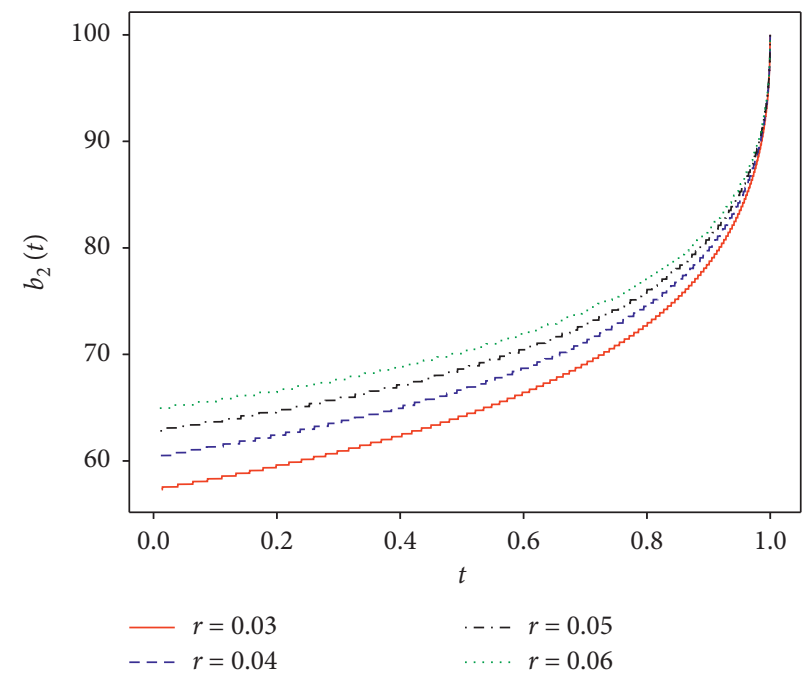

(b)

FIGURE 2: (a) The impact of varying interest rates on the optimal surrender boundary for regime 1; (b) the impact of varying interest rates on the optimal surrender boundary for regime 2 .

$(0.25,0.3)$, respectively. We note from this figure that the surrender boundaries move down as volatility increases.

We note that surrender happens only when the discounted fund value falls below the optimal surrender boundary. Hence, intuitively, for Figures 1 and 6, because the embedded guarantee with surrender option is a kind of American put option, the higher volatility means that the option is more valuable, which leads to a lower surrender boundary; for Figures 2-5, a higher $r$ or a higher $\kappa$ or a lower $c$ or a higher $G$ will provide a greater payoff to the policyholder and enlarge the surrender region. In conclusion, all the above cases are consistent with the theoretical findings in Theorems 1, 4, and 5.
Example 2. In this example, we depict sample path of the discounted mutual fund value $Y_{t}=e^{-\kappa(T-t)} F_{t}$ and the corresponding optimal surrender boundary in Figure 7 . The time step is set to be 10,000 . Assume that the generator of the Markov chain is given by

$$
A=\left(\begin{array}{cc}
-0.4 & 0.4 \\
0.5 & -0.5
\end{array}\right) \text {. }
$$

We set the initial fund value to be $F_{0}=100$ and the initial state of Markov chain $\alpha_{0}=1$. Also, we suppose the minimum guarantee amount $G=100$, the interest rate $r=0.06$, the insurance fee in percentage $c=0.02$, the penalty rate $\kappa=0.01$, and the volatility $\left(\sigma_{1}, \sigma_{2}\right)=(0.25,0.45)$. From this figure, we see that when the discounted fund value falls to 


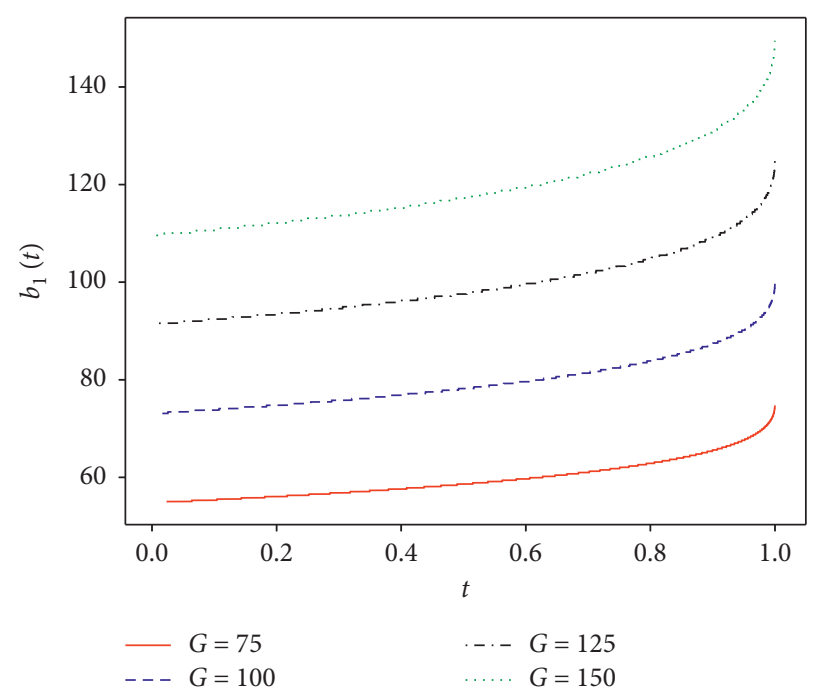

(a)

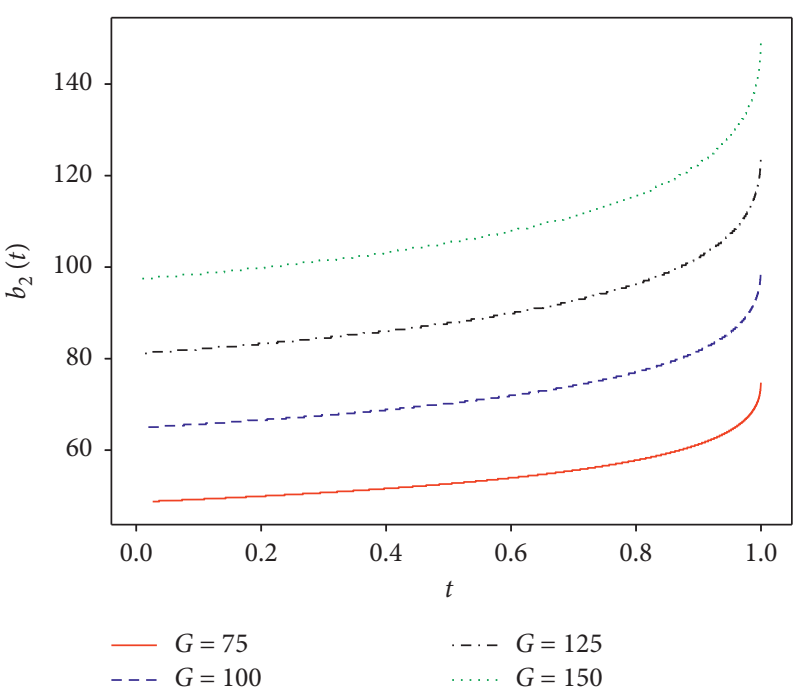

(b)

FIGURE 3: (a) The impact of varying minimum guarantee levels on the optimal surrender boundary for regime 1; (b) the impact of varying minimum guarantee levels on the optimal surrender boundary for regime 2 .

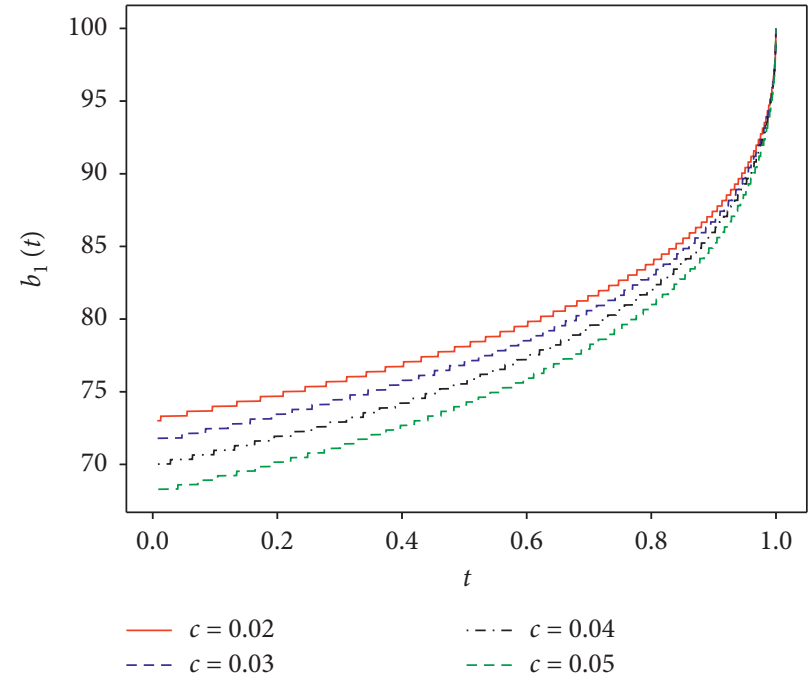

(a)

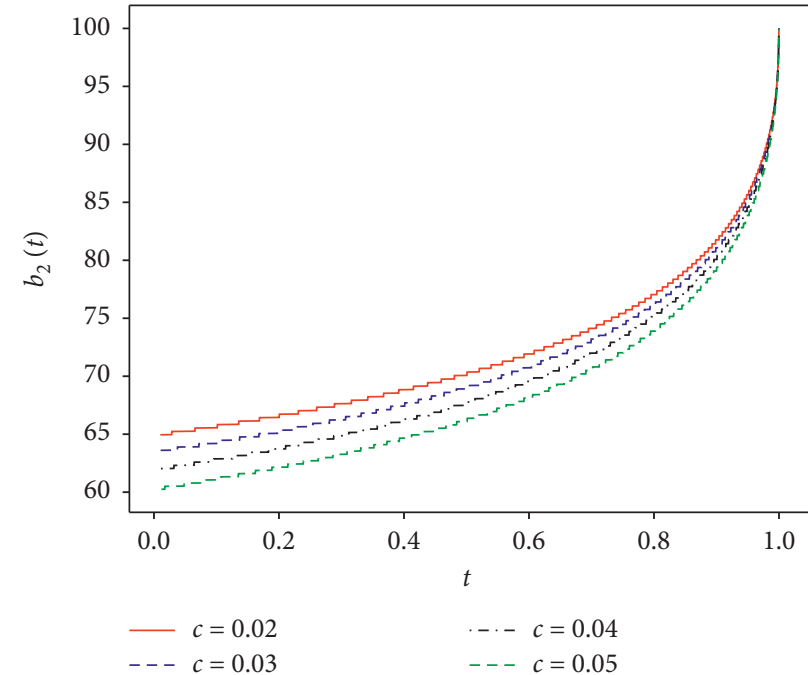

(b)

FIGURE 4: (a) The impact of varying fee rates on the optimal surrender boundary for regime 1; (b) the impact of varying fee rates on the optimal surrender boundary for regime 2 .

$Y_{t_{0}}$, the state of the economy switches to $\alpha_{t_{0}}=2$. The sample path of the discounted fund value hits the optimal surrender boundary in regime 2 for the first time. Hence, the policyholder should surrender to receive minimum benefit.

\section{Proofs}

In this section, we give detailed proofs of the results in the paper. 


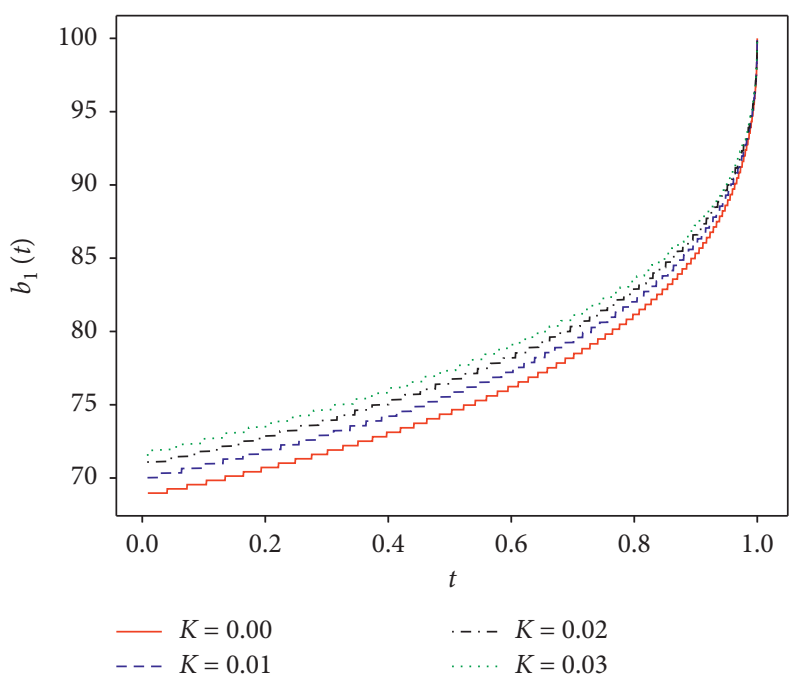

(a)

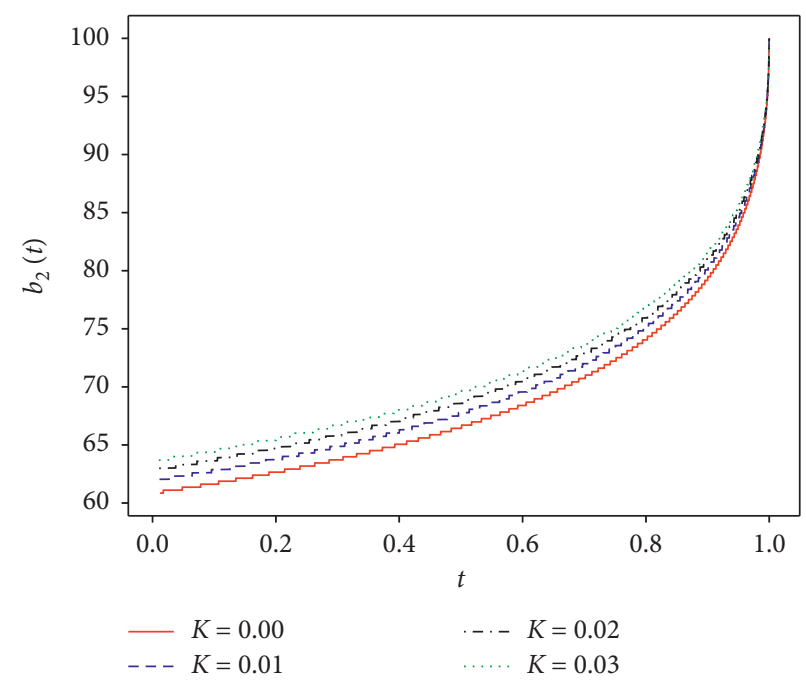

(b)

FIGURE 5: (a) The impact of varying penalty rates on the optimal surrender boundary for regime 1; (b) the impact of varying penalty rates on the optimal surrender boundary for regime 2 .

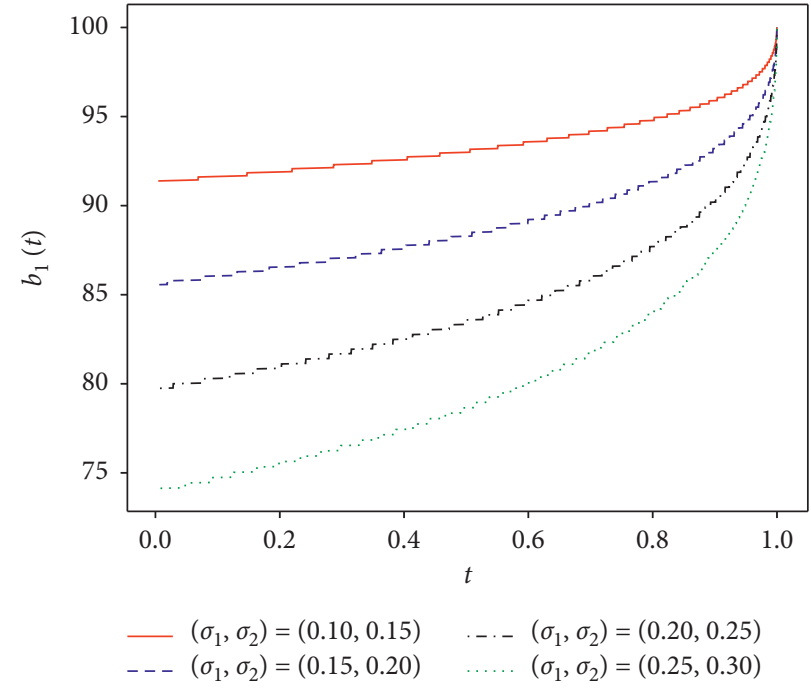

(a)

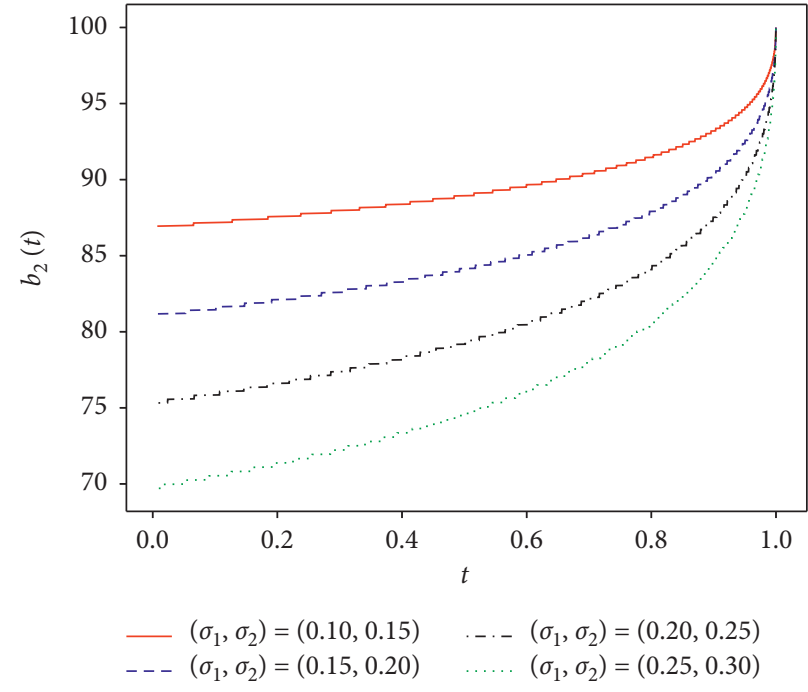

(b)

FIgURE 6: (a) The impact of varying volatility on the optimal surrender boundary for regime 1; (b) the impact of varying volatility on the optimal surrender boundary for regime 2 . 

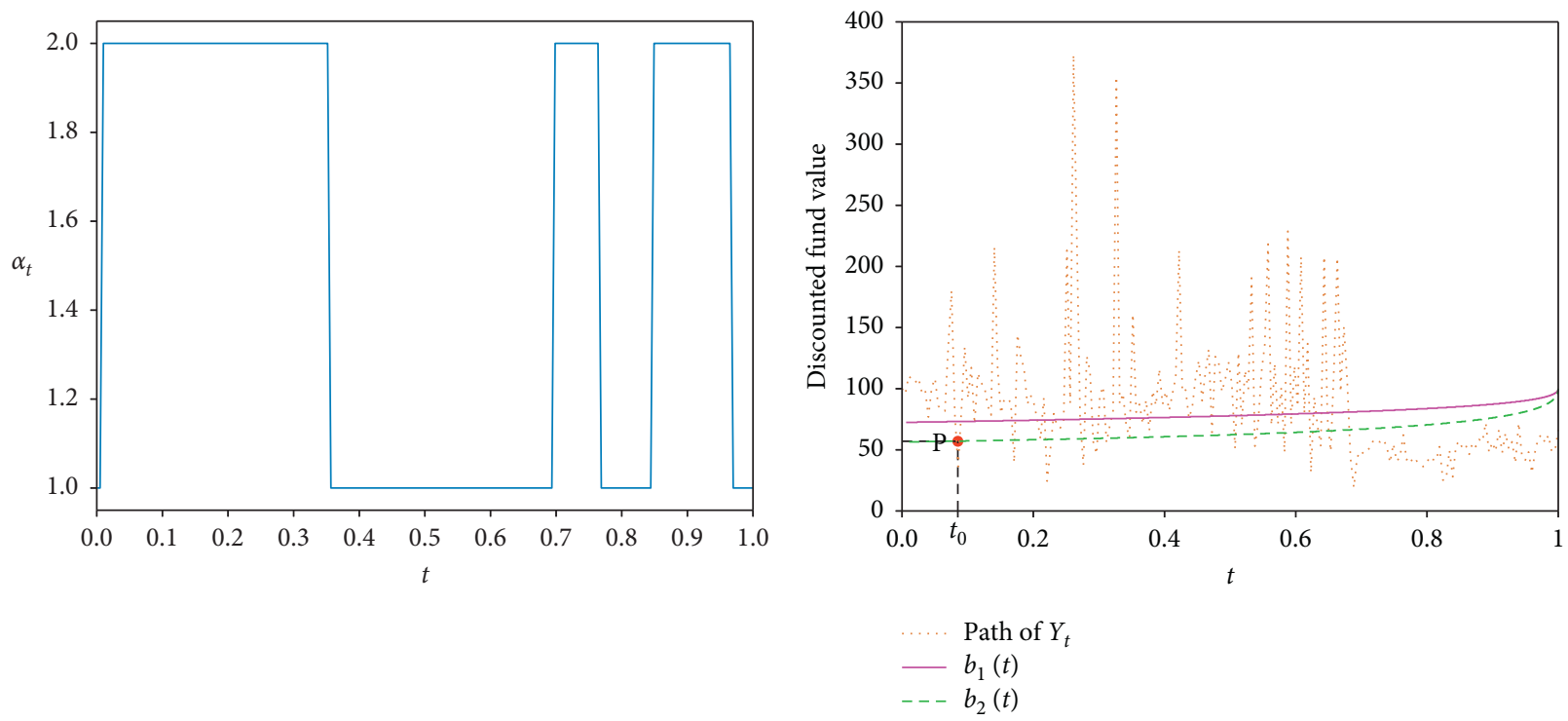

(a)

(b)

Figure 7: (a) Sample path of continuous time Markov chain with initial state $\alpha_{0}=1$; (b) optimal surrender boundary and sample path of fund value.

\subsection{Proof of Lemma 3}

Proof. For any $y_{1}, y_{2}>0$, by the definition of $V$ (see (15)) and moment estimates of $M_{t}$ (see [19]), we have

$$
\begin{aligned}
\left|V\left(t, y_{1}, i\right)-V\left(t, y_{2}, i\right)\right| & =\left|\sup _{0 \leq \tau \leq T-t} \mathbb{E}\left[e^{-r \tau} h\left(Y_{\tau}^{y_{1}}\right)\right]-\sup _{0 \leq \tau \leq T-t} \mathbb{E}\left[e^{-r \tau} h\left(Y_{\tau}^{y_{2}}\right)\right]\right| \\
& \leq \sup _{0 \leq \tau \leq T-t}\left|\mathbb{E}\left[e^{-r \tau}\left(h\left(Y_{\tau}^{y_{1}}\right)-h\left(Y_{\tau}^{y_{2}}\right)\right)\right]\right| \\
& \leq \sup _{0 \leq \tau \leq T-t} \mathbb{E}\left[\left|Y_{\tau}^{y_{1}}-Y_{\tau}^{y_{2}}\right|\right] \\
& \leq \sup _{0 \leq \tau \leq T-t}\left|y_{1}-y_{2}\right| \mathbb{E}\left[\left|M_{\tau}\right|\right] \\
& \leq C\left|y_{1}-y_{2}\right|,
\end{aligned}
$$

which means that $V$ is Lipschitz continuous in $y$.

Next, we assume that $t_{1}<t_{2}$ and $\tau_{1}$ is an $\varepsilon$-optimal stopping time for $V\left(t_{1}, y, i\right)$. Setting $\tau_{2}=\tau_{1} \wedge\left(T-t_{2}\right)$, we obtain that

$$
\begin{aligned}
0 & \leq V\left(t_{1}, y, i\right)-V\left(t_{2}, y, i\right) \\
& \leq \mathbb{E}\left[e^{-r \tau_{1}} h\left(Y_{\tau_{1}}^{y}\right)\right]+\varepsilon-\mathbb{E}\left[e^{-r \tau_{2}} h\left(Y_{\tau_{2}}^{y}\right)\right] \\
& \leq \mathbb{E}\left[e^{-r \tau_{2}}\left(h\left(Y_{\tau_{1}}^{y}\right)-h\left(Y_{\tau_{2}}^{y}\right)\right)\right]+\varepsilon \leq \mathbb{E}\left[\left|Y_{\tau_{1}}^{y}-Y_{\tau_{2}}^{y}\right|\right]+\varepsilon .
\end{aligned}
$$

Integrating both sides of (13) and applying Burkholder-Davis-Gundy inequality, we derive that 


$$
\begin{aligned}
\mathbb{E}\left[\left|Y_{\tau_{1}}^{y}-Y_{\tau_{2}}^{y}\right|\right] & \leq \mathbb{E}\left[\left|\int_{\tau_{2}}^{\tau_{1}}(r-c+\kappa) Y_{s} \mathrm{~d} s\right|\right]+\mathbb{E}\left[\left|\int_{\tau_{2}}^{\tau_{1}} \sigma_{s} Y_{s} \mathrm{~d} W_{s}\right|\right] \\
& \leq \mathbb{E}\left[\left|\int_{0}^{\tau_{1}-\tau_{2}}(r-c+\kappa) Y_{s+\tau_{2}} \mathrm{~d} s\right|\right]+\mathbb{E}\left[\left|\int_{0}^{\tau_{1}-\tau_{2}} \sigma_{s+\tau_{2}} Y_{s+\tau_{2}} \mathrm{~d} W_{s}\right|\right] \\
& \leq(r-c+\kappa) \mathbb{E}\left[\sup _{0 \leq t \leq T}\left|Y_{t}\right|\right]\left(t_{2}-t_{1}\right)+\mathbb{E}\left[\sup _{0 \leq t \leq t_{2}-t_{1}}\left|\int_{0}^{t} \sigma_{s+\tau_{2}} Y_{s+\tau_{2}} \mathrm{~d} W_{s}\right|\right] \\
& \leq(r-c+\kappa) \mathbb{E}\left[\sup _{0 \leq t \leq T}\left|Y_{t}\right|\right]\left(t_{2}-t_{1}\right)+C^{\prime} \mathbb{E}\left[\sqrt{\left.\int_{0}^{t_{2}-t_{1}} \sigma_{s+\tau_{2}}^{2} Y_{s+\tau_{2}}^{2} \mathrm{~d} s\right]}\right. \\
& \leq(r-c+\kappa) \mathbb{E}\left[\sup _{0 \leq t \leq T}\left|Y_{t}\right|\right]\left(t_{2}-t_{1}\right)+C^{\prime}\left(\sigma_{1}+\sigma_{2}\right) \mathbb{E}\left[\sup _{0 \leq t \leq T}\left|Y_{t}\right|\right] \sqrt{t_{2}-t_{1}},
\end{aligned}
$$

where $\sigma_{s}$ means $\sigma\left(\alpha_{s}\right)$. By moment estimates (see [19]), we have $\mathbb{E}\left[\sup _{0 \leq t \leq T}\left|Y_{t}\right|\right] \leq C^{\prime \prime}$. Letting $\varepsilon \longrightarrow 0$, we conclude that $V$ is $1 / 2$-Hölder continuous in $t$.

\subsection{Proof of Lemma 4}

Proof. Assume that $y_{1}<y_{2}$ and let $\tau$ be the optimal stopping time for $V\left(t, y_{1}, i\right)$. By the definition of $V$ (see (15)) and the assumption that $\kappa<c$, we derive that

$$
\begin{aligned}
V\left(t, y_{2}, i\right)-V\left(t, y_{1}, i\right) & \geq \mathbb{E}\left[e^{-r \tau} h\left(Y_{\tau}^{y_{2}}\right)\right]-\mathbb{E}\left[e^{-r \tau} h\left(Y_{\tau}^{y_{1}}\right)\right] \\
& \geq \mathbb{E}\left[e^{-r \tau}\left(Y_{\tau}^{y_{1}}-Y_{\tau}^{y_{2}}\right)\right] \\
& =\left(y_{1}-y_{2}\right) \mathbb{E}\left[e^{(\kappa-c) \tau} N_{\tau}\right] \\
& \geq y_{1}-y_{2},
\end{aligned}
$$

where $N_{t}=\exp \left(\int_{0}^{t}-(1 / 2) \sigma_{s}^{2} \mathrm{~d} s+\int_{0}^{t} \sigma_{s} \mathrm{~d} W_{s}\right)$ is a martingale. This implies the monotonicity of the function $y \mapsto V(t, y, i)+y$. If $\left(t, y^{\prime}, i\right) \in \mathscr{D}$, then the above inequality leads to

$$
\begin{aligned}
0 \leq & V(t, y, i)-h(y) \leq V(t, y, i) \\
& -(G-y) \leq V\left(t, y^{\prime}, i\right)-\left(G-y^{\prime}\right)=0, \quad \text { for } y<y^{\prime},
\end{aligned}
$$

that is, $(t, y, i) \in \mathscr{D}$.

\subsection{Proof of Theorem 1}

Proof. We make an assertion that

$$
\mathscr{D}_{i} \subset\{(t, y): y \leq G\} \cap\left\{(t, y): y \leq \frac{r G}{(c-\kappa)}\right\} .
$$

For any $(t, y) \in \mathscr{D}_{i}$, since $V>0$ (Lemma 2) and $V(t, y, i)=(G-y)^{+}$, we derive that $y<G$.

We denote $g(y):=G-y$. By Dynkin's formula, we obtain that
For any $(t, y) \in\{(t, y):-r g(y)+(\mathscr{L} g)(t, y, i)>0\}$, we can choose an open neighborhood $U \subset[0, T] \times(0,+\infty)$ of $(t, y)$ such that

$$
-r g(y)+(\mathscr{L} g)(t, y, i)>0
$$

for any $(t, y) \in U$. Let $\tau_{U}$ be the first exist time of $U$ when $\left(t+s, Y_{s}^{y}\right)$ started at $(t, y)$ at time 0 . Denote by $T_{i}$ the first transition time of $\alpha$ with $\alpha(0)=i$. Let $\tau^{*}:=\tau_{U} \wedge T_{i} \wedge T-t$. Since $-r g\left(Y_{v}^{y}\right)+(\mathscr{L} g)\left(t+v, Y_{v}^{y}, \alpha_{v}\right)>0$ for $v \in\left(0, \tau^{*}\right)$ a.s., setting $s=\tau^{*}$ in (54), by (15), we derive that

$$
\begin{aligned}
V(t, y, i) & \geq \mathbb{E}\left[e^{-r \tau^{*}} h\left(Y_{\tau^{*}}^{y}\right)\right] \geq \mathbb{E}\left[e^{-r \tau^{*}} g\left(Y_{\tau^{*}}^{y}\right)\right] \\
& =g(y)+\mathbb{E}\left[\int_{0}^{\tau^{*}} e^{-r v} \cdot(-r g+\mathscr{L} g)\left(t+v, Y_{v}^{y}, \alpha_{v}\right) \mathrm{d} v\right] \\
& >g(y) .
\end{aligned}
$$

This implies that $(t, y) \in \mathscr{C}_{i}$, which is equivalent to

$$
\mathscr{D}_{i} \subset\{(t, y):-r g(y)+(\mathscr{L} g)(t, y, i) \leq 0\}=\left\{(t, y): y \leq \frac{r G}{(c-\kappa)}\right\} \text {. }
$$

Moreover, $\mathscr{D}_{i} \neq \varnothing$. In fact, by Lemma 1 ,

$$
V_{t}(t, y, i)+(\mathscr{A} V)(t, y, i)-r V(t, y, i)=0 \text { in } \mathscr{C}_{i} \text {. }
$$

If $\mathscr{D}_{i}=\varnothing$, then $\mathscr{C}_{i}=[0, T] \times(0,+\infty)$. Letting $t \longrightarrow T$,

$$
\begin{aligned}
\lim _{t \longrightarrow T} V(t, y, i) & =r h(y)-(\mathscr{L} h)(t, y) \\
& =r G-(c-\kappa) y>0,
\end{aligned}
$$

when $y<r G /(c-\kappa)$. This contradicts with the fact that $V$ is nonincreasing in $t$ (Lemma 2). Now, the downward connectedness implies (22).

Then, we shall discuss the monotonicity of $b_{i}(t)$. Assume that there exists some $0 \leq t_{1} \leq t_{2} \leq T$ such that $b_{i}\left(t_{1}\right)>b_{i}\left(t_{2}\right)$, by the definition of $b_{i}(t)$ (see $(22)$ ), we derive that 


$$
V\left(t_{2}, b_{i}\left(t_{1}\right), i\right)>h\left(b_{i}\left(t_{1}\right)\right)=V\left(t_{1}, b_{i}\left(t_{1}\right), i\right),
$$

which is a contradiction (Lemma 2).

Next, we consider the continuity of $b_{i}(t)$. Suppose that there exists some $t_{0} \in[0, T)$ such that

$$
\lim _{t \rightarrow t_{0}^{-}} b_{i}(t)=y_{1}<\lim _{t \longrightarrow t_{0}^{+}} b_{i}(t)=y_{2} .
$$

Letting $t_{n} \geq t_{0}$ and $t_{n} \longrightarrow t_{0}$ as $n \longrightarrow+\infty$, obviously, $\left(t_{n}, b_{i}\left(t_{n}\right)\right) \in \mathscr{D}_{i}$. Since $\mathscr{D}_{i}$ is closed, we have $\left(t_{0}, b_{i}\left(t_{0}+\right)\right) \in \mathscr{D}_{i}$. By the definition of $b_{i}, b_{i}\left(t_{0}\right) \geq b_{i}\left(t_{0}+\right)$. On the contrary, since $b_{i}$ is increasing, $b_{i}\left(t_{0}+\right) \geq b_{i}\left(t_{0}\right)$. Hence, $b_{i}(t)$ is right continuous, i.e., $\left(t_{0}, y\right) \in \mathscr{D}_{i}$, for $y_{1}<y<y_{2}$.

By Lemma 1 , for any $(t, y, i) \in(0, T) \times(0,+\infty) \times \mathcal{S}$,

$$
\left(V_{t}+\mathscr{A} V-r V\right)(t, y, i) \leq 0 .
$$

Letting $t \longrightarrow t_{0}+$, then, for any $y \in\left(y_{1}, y_{2}\right)$,

$$
r V\left(t_{0}, y, j\right) \leq \frac{1}{\lambda_{i}}\left[\left(r+\lambda_{i}\right) G-\left(c-\kappa+\lambda_{i}\right) y\right] .
$$

On the contrary, letting $t \longrightarrow t_{0}$, in (58), we derive that

$$
\begin{aligned}
V\left(t_{0}, y, j\right) & =\frac{1}{\lambda_{i}}\left[\left(r+\lambda_{i}\right) V\left(t_{0}, y, i\right)-\left(V_{t}+\mathscr{L} V\right)\left(t_{0}, y, i\right)\right] \\
& \geq \frac{1}{\lambda_{i}}\left[\left(r+\lambda_{i}\right) G-\left(c-\kappa+\lambda_{i}\right) y\right],
\end{aligned}
$$

where the last inequality follows from Lemma 2. Therefore,

$$
V\left(t_{0}, y, j\right)=\frac{1}{\lambda_{i}}\left[\left(r+\lambda_{i}\right) G-\left(c-\kappa+\lambda_{i}\right) y\right] .
$$

This leads to

$$
V_{y}\left(t_{0}, y, j\right)=-\frac{c-\kappa+\lambda_{i}}{\lambda_{i}}<-1,
$$

where the last inequality follows from the fact that $c>\kappa$. This contradicts with Lemma 4 . Hence, $b_{i}(t)$ is continuous.
Finally, we prove (23) holds. As $b_{i}(t)$ is nondecreasing and (53) holds, we have $b_{i}(T)$ is well defined and $b_{i}(T):=\lim _{t \longrightarrow T^{-}} b_{i}(t) \leq \min \{G, r G /(c-\kappa)\}:=y^{*}$. Suppose that $b_{i}(T)<y^{*}$. For any $\bar{y} \in\left(b_{i}(T), y^{*}\right)$, since $V(T, y, i)=h(y)=g(y)$, letting $t \longrightarrow T^{-}$in (58), we have

$$
V_{t}(T, \bar{y}, 1)=r V(T, \bar{y}, 1)-(\mathscr{A} V)(T, \bar{y}, 1)=r G-(c-\kappa) \bar{y}>0,
$$

which contradicts with $V_{t} \leq 0$ (Lemma 2).

\subsection{Proof of Theorem 2}

Proof. (24) follows directly from (58). (25) follows from the continuity of $V$ (Lemma 3). (27) follows from (15) directly.

We shall prove the smooth pasting condition (26). We set $y=b_{i}(t)$. As $(t, y+\varepsilon) \in \mathscr{C}_{i}$, we derive that

$$
\frac{V(t, y+\varepsilon, i)-V(t, y, i)}{\varepsilon} \geq \frac{G-y-\varepsilon-(G-y)}{\varepsilon}=-1,
$$

where the inequality follows from (15) and (25) and $V$ is positive. Letting $\varepsilon \longrightarrow 0$,

$$
V_{y}^{+}(t, y, i) \geq-1 \text {, }
$$

where $V_{y}^{+}$is the right derivative of $V$ in $y$. For any $\varepsilon>0$, let $\tau_{\varepsilon}^{\prime}$ be the optimal stopping time for $V(t, y+\varepsilon, i)$ and $T_{i}$ be the first transition time of $\alpha$ with $\alpha(0)=i$. Denote

$$
\tau_{\varepsilon}:=\inf \left\{s \in\left[0, T_{i}\right] \mid Y_{s}^{y+\varepsilon} \leq b_{i}(t+s)\right\} .
$$

Obviously, if $T_{i} \geq T-t$, then $\tau_{\varepsilon}^{\prime}=\tau_{\varepsilon}$; otherwise, we have

$$
\begin{aligned}
\tau_{\varepsilon}^{\prime}(t, y, i) & =\inf \left\{s \in[0, T-t] \mid Y_{s}^{y+\varepsilon} \leq b_{\alpha_{s}}(t+s)\right\} \\
& =\tau_{\varepsilon} \wedge \inf \left\{s \in\left[T_{i}, T-t\right] \mid Y_{s}^{y+\varepsilon} \leq b_{\alpha_{s}}(t+s)\right\} .
\end{aligned}
$$

By (14), we have

$$
\begin{aligned}
\tau_{\varepsilon} & =\inf \left\{s \in\left[0, T_{i}\right]: \int_{0}^{s}\left(r-c+\kappa-\frac{1}{2} \sigma^{2}\left(\alpha_{v}\right)\right) \mathrm{d} v+\int_{0}^{s} \sigma\left(\alpha_{v}\right) \mathrm{d} W_{v} \leq \log \left(\frac{b_{i}(t+s)}{y+\varepsilon}\right)\right\} \\
& \leq \inf \left\{s \in\left[0, T_{i}\right]:\left(r-c+\kappa-\frac{1}{2} \sigma_{i}^{2}\right) s+\sigma_{i} W_{s} \leq \log \left(\frac{y}{y+\varepsilon}\right)\right\},
\end{aligned}
$$

where the inequality follows from $b_{i}$ which is nondecreasing.

By the law of the iterated logarithm, $\mathbb{Q}\left[\min _{0 \leq t \leq a}(r-\right.$ $\left.\left.c+\kappa-(1 / 2) \sigma_{i}^{2}\right) s+\sigma_{i} W_{s}<0\right]=1$. This means that, for any $a>0$, there exists some $\varepsilon>0$ small enough such that $\tau_{\varepsilon}<a$, i.e., $\tau_{\varepsilon} \longrightarrow 0$ as $\varepsilon \longrightarrow 0$. Hence, (71) leads to $\tau_{\varepsilon}^{\prime} \longrightarrow 0$ as $\varepsilon \longrightarrow 0$. 
Now, we prepare to prove $V_{y}^{+}(t, y, i) \leq-1$. As $\tau_{\varepsilon}^{\prime}$ is the first time that $Y_{t}^{y+\varepsilon}$ hits the free boundary and $b_{i} \leq G$, we have $G-Y_{\tau_{\varepsilon}^{\prime}}^{y+\varepsilon}>0$ and $G-Y_{\tau_{\varepsilon}^{\prime}}^{y}>0$. Hence,

$$
\begin{aligned}
\frac{V(t, y+\varepsilon, i)-V(t, y, i)}{\varepsilon} & \leq \frac{\mathbb{E}\left[e^{-r \tau_{\varepsilon} \prime}\left(G-Y_{\tau_{\varepsilon}^{\prime}}^{y+\varepsilon}\right)\right]-\mathbb{E}\left[e^{-r \tau_{\varepsilon} \prime}\left(G-Y_{\tau_{\varepsilon}^{\prime}}^{y}\right)\right]}{\varepsilon} \\
& =\frac{\mathbb{E}\left[e^{-r \tau_{\varepsilon} \prime}\left(Y_{\tau_{\varepsilon}^{\prime}}^{y}-Y_{\tau_{\varepsilon}^{\prime}}^{y+\varepsilon}\right)\right]}{\varepsilon} \\
& =-\mathbb{E}\left[M_{\tau_{\varepsilon}^{\prime}}\right]
\end{aligned}
$$

where $M_{t}$ is defined in (14). Letting $\varepsilon \longrightarrow 0$, by Fatou's lemma, we conclude that $V_{y}^{+}(t, y, i)=-1$. As $V(t, y, i)=$ $G-y$ for $y<b_{i}(t)$, it is easy to see that $V_{y}^{-}(t, y, i)=-1$. Here, $V_{y}^{-}$is the left derivative of $V$ in $y$. We conclude that the smooth pasting condition (26) holds.

For uniqueness, let $f(t, y, i)$ defined on $\overline{\mathscr{C}}$ be a solution of the free boundary problem. We define $N_{s}:=e^{-r s} f(s+$ $\left.t, Y_{s}, \alpha_{s}\right)$ and set $\tau_{\mathscr{D}}:=\inf \left\{s \in[0, T-t]: Y_{s}^{y} \leq b_{\alpha_{s}}(s+t)\right\}$. By It $\widehat{o}$ 's lemma,

$$
\begin{aligned}
N_{s \wedge \tau_{\mathscr{D}}}= & f(t, y, i) \\
& +\int_{0}^{s \wedge \tau_{\mathscr{D}}} e^{-r v}\left(f_{t}+\mathscr{A} f-r f\right)\left(t+v, Y_{v}^{y}, \alpha_{v}\right) \mathrm{d} v+\tilde{N}_{s \wedge \tau_{\mathscr{D}}},
\end{aligned}
$$

where $\left\{\tilde{N}_{s \wedge \tau_{D}}, s \geq 0\right\}$ is a martingale. Hence, by (24), $\left\{N_{s \wedge \tau_{D}}\right.$, $s \geq 0\}$ is a martingale. We know from the optional sampling theorem that

$$
\begin{aligned}
f(t, y, i) & =N_{0}=\mathbb{E}\left[N_{\tau_{\mathscr{D}}}\right] \\
& =\mathbb{E}\left[e^{-r \tau_{\mathscr{D}}} f\left(\tau_{\mathscr{D}}+t, Y_{\tau_{\mathscr{D}}}, \alpha_{\tau_{\mathscr{D}}}\right)\right] \\
& =\mathbb{E}\left[e^{-r \tau_{\mathscr{D}}} h\left(Y_{\tau_{\mathscr{D}}}\right)\right] \\
& =V(t, y, i),
\end{aligned}
$$

where the second equality follows from that $\left\{N_{s \wedge \tau_{D}}, s \geq 0\right\}$ is a bounded martingale and dominated convergent theorem, the fourth equality follows from (25) and definition of $\tau_{\mathscr{D}}$, and the last equality follows from that $\tau_{\mathscr{D}}$ is an optimal stopping time of $V(t, y, i)$. This implies the uniqueness.

By Lemma 3 and regularity of parabolic equation (see [20]), it is obvious that $V_{t}, V_{y}$, and $V_{y y}$ exist and are continuous in $\mathscr{C}$.

\subsection{Proof of Theorem 3}

Proof. Firstly, we consider the following optimal stopping problem:

$$
V_{i}(t, y)=\sup _{0 \leq \tau \leq T-t} \mathbb{E}\left[e^{-\left(r+\lambda_{i}\right) \tau h}\left(Y_{\tau}^{(i)}\right)+\lambda_{i} \int_{0}^{\tau} e^{-\left(r+\lambda_{i}\right) u} V\left(t+u, Y_{u}^{(i)}, j\right)\right], \quad j \neq i
$$


where $V(t, y, i)$ is defined in (15) and $Y_{t}^{(i)}$ satisfies (31). Fixing $i \in \mathcal{S}$, by a similar Markovian argument, we conclude that $V_{i}(t, y)$ is a solution to the free boundary problems (24)-(27). The uniqueness implies that $V_{i}(t, y)=V(t, y, i)$ for $(t, y, i) \in[0, T] \times(0,+\infty) \times \mathcal{S}$.
By the regularity of $V$ (see Theorem 2), we can use the local time-space formula given in Peskir and Shiryaev [18] to obtain that

$$
\begin{aligned}
e^{-\left(r+\lambda_{i}\right) s} V_{i}\left(s+t, Y_{s}^{(i)}\right)= & \left.V_{i}(t, y)+\int_{0}^{s} e^{-\left(r+\lambda_{i}\right) u}\left(V_{i t}+\frac{1}{2} \sigma_{i}^{2} y^{2} V_{i y y}+(r-c+\kappa) y V_{i y}-\left(r+\lambda_{i}\right) V_{i}\right)\left(t+u, Y_{u}^{(i)}\right) \chi_{\left\{Y_{u}^{(i)} \neq b_{i}(u+t)\right\}} \mathrm{d} u\right) \\
& +\int_{0}^{s} \sigma_{i} Y_{u}^{(i)} V_{i y}\left(t+u, Y_{u}^{(i)}\right) \chi_{\left\{Y_{u}^{(i)} \neq b_{i}(u+t)\right\}} \mathrm{d} W_{u} \\
& +\frac{1}{2} \int_{0}^{s}\left(V_{i y}\left(t+u, Y_{u}^{(i)}+\right)-V_{i y}\left(t+u, Y_{u}^{(i)}-\right)\right) \cdot \chi_{\left\{Y_{u}^{(i)}=b_{i}(u+t)\right\}} \mathrm{d} \ell_{u}^{b_{i}}\left(Y^{(i)}\right),
\end{aligned}
$$

where $\ell_{u}^{b_{i}}\left(Y^{(i)}\right)$ is the local time of $Y^{(i)}$ at the curve $b_{i}$ given by

$$
\ell_{u}^{b_{i}}\left(Y^{(i)}\right):=\lim _{\varepsilon \longrightarrow 0} \frac{1}{2 \varepsilon} \int_{0}^{u} \chi_{\left\{b_{i}(v)-\varepsilon<Y_{v}^{(i)}<b_{i}(v)+\varepsilon\right\}} \mathrm{d}\left\langle Y^{(i)}\right\rangle_{v},
$$

and $\mathrm{d} \ell_{u}^{b_{i}}\left(Y^{(i)}\right)$ refers to integration with respect to the continuous increasing function $u \mapsto \ell_{u}^{b_{i}}\left(Y^{(i)}\right)$.

We notice that $V_{i}(t, y)=G-y$ for $y<b_{i}(t)$ and $V_{i}$ satisfies (58) for $y>b_{i}(t)$. Hence,

$$
\left(V_{i t}+\frac{1}{2} \sigma_{i}^{2} y^{2} V_{i y y}+(r-c+\kappa) y V_{i y}-\left(r+\lambda_{i}\right) V_{i}\right)\left(t+u, Y_{u}^{(i)}\right)= \begin{cases}\Phi_{i}\left(Y_{u}^{(i)}\right), & \text { if } Y_{u}^{(i)}<b_{i}(u+t), \\ -\lambda_{i} V\left(u, Y_{u}^{(i)}, j\right), & \text { if } Y_{u}^{(i)}>b_{i}(u+t),\end{cases}
$$

where $j \neq i$. Setting $s=T-t$ together with (26) and (27), taking expectation in (77), and using notations (28) and (29), we derive that

$$
\begin{aligned}
V_{i}(t, y) & =\mathbb{E}\left[e^{-\left(r+\lambda_{i}\right)(T-t)} h\left(Y_{T-t}^{(i)}\right)\right]-\mathbb{E}\left[\int_{0}^{T-t} e^{-\left(r+\lambda_{i}\right) u}\left(\Phi_{i}\left(Y_{u}^{(i)}\right) \chi_{\left\{Y_{u}^{(i)}<b_{i}(u+t)\right\}}-\lambda_{i} V\left(t+u, Y_{u}^{(i)}, j\right) \chi_{\left\{Y_{u}^{(i)}>b_{i}(u+t)\right\}}\right) \mathrm{d} u\right. \\
& =J_{i}(t, y)-\int_{0}^{T-t} K_{i}\left(t, y, u, b_{i}(u+t)\right) \mathrm{d} u \\
& =V(t, y, i) .
\end{aligned}
$$

Finally, by condition (25), we derive that

$$
J_{i}\left(t, b_{i}(t)\right)-\int_{0}^{T-t} K_{i}\left(t, b_{i}(t), u, b_{i}(u+t)\right) \mathrm{d} u=h\left(b_{i}(t)\right), \quad i \in \mathcal{S} .
$$


Proof. We set $V(t, y, i):=V_{i}(t, y)$, for $i \in \mathcal{S}$. We denote $\mathcal{N}:=\left\{(t, y): V_{1}(t, y)<V_{2}(t, y)\right\}$. We shall prove $\mathcal{N}=\varnothing$. Otherwise, in $\mathcal{N}$,

$$
\begin{gathered}
V_{2}(t, y)>V_{1}(t, y) \geq h(y), \\
V_{2 t}+\frac{1}{2} \sigma_{2}^{2} y^{2} V_{2 y y}+(r-c+\kappa) y V_{2 y}-r V_{2}=-\lambda_{2}\left(V_{1}-V_{2}\right) \geq 0, \\
V_{1 t}+\frac{1}{2} \sigma_{1}^{2} y^{2} V_{1 y y}+(r-c+\kappa) y V_{1 y}-r V_{1}=-\lambda_{1}\left(V_{2}-V_{1}\right) \leq 0,
\end{gathered}
$$

where the last two inequalities follow from (58). By Lemma 2 , it follows that

$$
\begin{aligned}
& V_{1 t}+\frac{1}{2} \sigma_{2}^{2} y^{2} V_{1 y y}+(r-c+\kappa) y V_{1 y}-r V_{1} \\
& =V_{1 t}+\frac{1}{2} \sigma_{1}^{2} y^{2} V_{1 y y}+(r-c+\kappa) y V_{1 y}-r V_{1}+\frac{1}{2} y^{2}\left(\sigma_{2}^{2}-\sigma_{1}^{2}\right) V_{1 y y} \leq 0 .
\end{aligned}
$$

Furthermore, $V_{1}(T, y)=V_{2}(T, y)$ and $V_{1}=V_{2}$ on the boundary of $\mathcal{N}$. Hence, the comparison principle of variational inequality implies that $V_{1} \geq V_{2}$ in $\mathcal{N}$, which contradicts with definition of $\mathcal{N}$. This leads to $V_{1} \geq V_{2}$ for $(t, y) \in(0, T) \times(0,+\infty)$. That means $\mathscr{C}_{2} \subset \mathscr{C}_{1}$, i.e., $b_{2}(t) \geq b_{1}(t)$.

By Markovian argument, $V_{i}^{0}$ satisfies

$$
\begin{aligned}
\min \left\{-V_{i t}^{0}-\frac{1}{2} \sigma_{i}^{2} y^{2} V_{i y y}^{0}-(r-c+\kappa) y V_{i y}^{0}+r V_{i}^{0}, V_{i}^{0}-h(y)\right\} & =0 \\
V_{i}^{0}(T, y) & =h(y) .
\end{aligned}
$$

By Lemma 1, it follows that

$$
\begin{array}{r}
-V_{1 t}^{0}-\frac{1}{2} \sigma_{1}^{2} y^{2} V_{1 y y}^{0}-(r-c+\kappa) y V_{1 y}^{0}+r V_{1}^{0}-\lambda_{1}\left(V_{2}-V_{1}\right) \geq-\lambda_{1}\left(V_{2}-V_{1}\right) \geq 0, \\
-V_{2 t}-\frac{1}{2} \sigma_{2}^{2} y^{2} V_{2 y y}-(r-c+\kappa) y V_{2 y}+r V_{2} \geq \lambda_{2}\left(V_{1}-V_{2}\right) \geq 0 .
\end{array}
$$


Applying comparison principle of variational inequality, we deduce that $V_{1}^{0} \geq V_{1}$ and $V_{2} \geq V_{2}^{0}$, i.e., $b_{1}^{0}(t) \leq b_{1}(t)$ and $b_{2}(t) \leq b_{2}^{0}(t)$.

\subsection{Proof of Theorem 5}

Proof. Assume that $\left(\widehat{V}_{1}, \widehat{V}_{2}\right)$ is the solution to variational inequality (18), where $\sigma_{i}$ is $\widehat{\sigma}_{i},\left(\bar{V}_{1}, \bar{V}_{2}\right)$ is the solution to variational inequality (18), where $\sigma_{i}$ is $\bar{\sigma}_{i}$, and $\widehat{\sigma}_{i} \geq \bar{\sigma}_{i}$ for each $i \in \mathcal{S}$. By Lemmas 1 and 2 , it follows that

$$
\widehat{V}_{i t}+\frac{1}{2} \bar{\sigma}_{i}^{2} y^{2} \widehat{V}_{i y y}+(r-c+\kappa) y \widehat{V}_{i y}-r \widehat{V}_{i}+\lambda_{i}\left(\widehat{V}_{j}-\widehat{V}_{i}\right)=\frac{1}{2} y^{2}\left(\bar{\sigma}_{i}^{2}-\widehat{\sigma}_{i}^{2}\right) \widehat{V}_{i y y} \leq 0
$$

if $\widehat{V}_{i}(t, y)>h(y)$ and

$$
\widehat{V}_{i t}+\frac{1}{2} \bar{\sigma}_{i}^{2} y^{2} \widehat{V}_{i y y}+(r-c+\kappa) y \widehat{V}_{i y}-r \widehat{V}_{i}+\lambda_{i}\left(\widehat{V}_{j}-\widehat{V}_{i}\right) \leq \frac{1}{2} y^{2}\left(\bar{\sigma}_{i}^{2}-\widehat{\sigma}_{i}^{2}\right) \widehat{V}_{i y y} \leq 0
$$

if $\widehat{V}_{i}(t, y)=h(y)$. Employing comparison principle of the variational system (see [15]), we conclude that $\widehat{V}_{i} \geq \bar{V}_{i}$, i.e., $\bar{b}_{i}(t) \geq \widehat{b}_{i}(t)$, for $i \in \mathcal{S}$. Hence, $\left(b_{1}(t), b_{2}(t)\right)$ is decreasing with respect to $\sigma_{i}$ for each $i \in \mathcal{S}$.

Next, assume that $\left(\widehat{V}_{1}, \widehat{V}_{2}\right)$ is the solution to variational inequality (18), where $G$ is $G_{1},\left(\bar{V}_{1}, \bar{V}_{2}\right)$ is the solution to variational inequality (18), where $G$ is $G_{2}$, and $G_{1}<G_{2}$. Then, we have

$$
\begin{aligned}
\bar{V}_{i}(t, y) & =\sup _{0 \leq \tau \leq T-t} \mathbb{E}^{i, y}\left[e^{-r \tau}\left(G_{2}-Y_{\tau}\right)^{+}\right] \\
& \leq G_{2}-G_{1}+\sup _{0 \leq \tau \leq T-t} \mathbb{E}^{i, y}\left[e^{-r \tau}\left(G_{1}-Y_{\tau}\right)^{+}\right] \\
& =G_{2}-G_{1}+\widehat{V}_{i}(t, y) .
\end{aligned}
$$

This implies that if $\bar{V}_{i}(t, y)>G_{2}-y$, then $\widehat{V}_{i}(t, y) \geq \bar{V}_{i}(t, y)+G_{1}-G_{2}>G_{1}-y$. If we denote by $\hat{\mathscr{C}}_{i}$ the stopping region with respect to $G_{1}$ and $\overline{\mathscr{C}}_{i}$ the stopping region with respect to $G_{2}$, then $\overline{\mathscr{C}}_{i} \subset \widehat{\mathscr{C}}_{i}$, i.e., $\bar{b}_{i} \geq \widehat{b}_{i}$. Hence, $\left(b_{1}(t), b_{2}(t)\right)$ is increasing with respect to $G$.

In the following, assume that $\left(\hat{V}_{1}, \widehat{V}_{2}\right)$ is the solution to variational inequality (18), where $\kappa$ is $\kappa_{1},\left(\bar{V}_{1}, \bar{V}_{2}\right)$ is the solution to variational inequality (18), where $\kappa$ is $\kappa_{2}$, and $\kappa_{1}<\kappa_{2}$. By Lemma 2, it follows that

$$
\widehat{V}_{i t}+\frac{1}{2} \sigma_{i}^{2} y^{2} \widehat{V}_{i y y}+\left(r-c+\kappa_{2}\right) y \widehat{V}_{i y}-r \widehat{V}_{i}+\lambda_{i}\left(\widehat{V}_{j}-\widehat{V}_{i}\right)=\left(\kappa_{2}-\kappa_{1}\right) y \widehat{V}_{i y} \leq 0
$$

if $\widehat{V}_{i}(t, y)>h(y)$ and

$$
\widehat{V}_{i t}+\frac{1}{2} \bar{\sigma}_{i}^{2} y^{2} \widehat{V}_{i y y}+\left(r-c+\kappa_{2}\right) y \widehat{V}_{i y}-r \widehat{V}_{i}+\lambda_{i}\left(\widehat{V}_{j}-\widehat{V}_{i}\right) \leq\left(\kappa_{2}-\kappa_{1}\right) y \widehat{V}_{i y} \leq 0
$$

if $\widehat{V}_{i}(t, y)=h(y)$. Employing comparison principle of the variational system (see [15]), we conclude that $\widehat{V}_{i} \geq \bar{V}_{i}$, i.e., $\bar{b}_{i}(t) \geq \widehat{b}_{i}(t)$, for $i \in \mathcal{S}$. By a similar argument, we can prove $\left(b_{1}(t), b_{2}(t)\right)$ is increasing with respect to $r$ and decreasing with respect to $c$.

\section{Conclusions}

In this paper, we study the valuation of the GMMB rider embedded in a VA contract. We focus on the optimal surrender boundary and use partial differential equation 
approach together with probabilistic approach to analyze the properties of it. Moreover, the impacts of the varying model parameters on the optimal surrender boundary are studied rigorously. The regime-switching Volterra-type integral equations are derived by the local time-space formula. However, the numerical methods to solve the integral equations remain unknown, which will be a challenging problem for future research. The numerical evidence supports our theoretical findings. Besides, it is important to extend our results by incorporating interest rates that depend on the Markov chain. We will consider this case in the future.

\section{Appendix}

\section{A. Proof of Lemma 1}

Proof. Firstly, we shall follow the steps in [18] to show that the value function $V(t, y, i)$ satisfies

$$
V_{t}(t, y, i)+(\mathscr{A} V)(t, y, i)-r V(t, y, i)=0 \text { in } \mathscr{C} .
$$

Define a killed process:

$$
\tilde{Y}_{t}:= \begin{cases}Y_{t}, & \text { if } t<T, \\ \Delta, & \text { if } t \geq T,\end{cases}
$$

where $T$ is a random variable exponentially distributed with parameter $r>0$ and independent of $Y$ and $\Delta$ is a fictitious point and all functions are assumed to take value zero at $\Delta$. By a direct computation, we have

$$
\begin{aligned}
\mathbb{E}\left[h\left(\widetilde{Y}_{t}\right)\right] & =\mathbb{E}\left[h\left(Y_{t}\right) \chi_{t<T}\right] \\
& =\mathbb{E}\left[h\left(Y_{t}\right) \chi_{t<T} \mid t<T\right] \mathbb{Q}(T>t)+\mathbb{E}\left[h\left(Y_{t}\right) \chi_{t<T} \mid t \geq T\right] \mathbb{Q}(T \leq t) \\
& =\mathbb{E}\left[e^{-r t} h\left(Y_{t}\right)\right]
\end{aligned}
$$

where the second equality follows from law of total expectation and the last equality follows from the fact that $Y_{t}$ is independent of $T$. Now, we can rewrite the value function as

$$
V(t, y, i)=\mathbb{E}\left[h\left(\widetilde{Y}_{\tau_{\mathscr{D}}}^{y}\right)\right] .
$$

We choose $(t, y, i) \in \mathscr{C}$ and a neighborhood $U \subset \mathscr{C}$ of $(t, y, i)$. Define $\sigma=\inf \left\{s \in[0, T-t]:\left(t+s, Y_{s}^{y}, \alpha_{s}\right) \notin U\right\}$. It is clear $\sigma \leq \tau_{\mathscr{D}}$. This leads to $\sigma+\tau_{\mathscr{D}} \circ \theta_{\sigma}=\tau_{\mathscr{D}}$ (see [18]). By strong Markov property,

$$
\begin{aligned}
\mathbb{E}\left[V\left(\sigma, \tilde{Y}_{\sigma}, \alpha_{\sigma}\right)\right] & =\mathbb{E}\left[\mathbb{E}\left[h\left(\widetilde{Y}_{\tau_{\mathscr{D}}}\right) \circ \theta_{\sigma} \mid \mathscr{F}_{\sigma}\right]\right] \\
& =\mathbb{E}\left[\mathbb{E}\left[h\left(\widetilde{Y}_{\sigma+\tau_{\mathscr{D}}} \circ \theta_{\sigma}\right) \mid \mathscr{F}_{\sigma}\right]\right] \\
& =\mathbb{E}\left[\mathbb{E}\left[h\left(\widetilde{Y}_{\tau_{\mathscr{D}}}\right) \mid \mathscr{F}_{\sigma}\right]\right] \\
& =\mathbb{E}\left[h\left(\widetilde{Y}_{\tau_{\mathscr{D}}}\right)\right] \\
& =V(t, y, i),
\end{aligned}
$$

where $\theta$ is the shift operator. This implies the characteristic operator of $\left(t, \widetilde{Y}_{t}, \alpha_{t}\right)$ is identically zero:

$$
\lim _{U \longrightarrow(t, y, i)} \frac{\mathbb{E}\left[V\left(\sigma, \widetilde{Y}_{\sigma}, \alpha_{\sigma}\right)\right]-V(t, y, i)}{\mathbb{E}[\sigma]}=0 .
$$

By the fact that the characteristic operator coincides with the infinitesimal generator of $\left(t, \widetilde{Y}_{t}, \alpha_{t}\right)$, we derive that (A.1) holds.

For any $\varepsilon>0$, letting $\tau_{\mathscr{D}}(t+\varepsilon, y, i)$ be an optimal stopping time for $V(t+\varepsilon, y, i)$, by a similar argument as (A.5), we have

$$
\begin{aligned}
\mathbb{E}\left[V\left(t+\varepsilon, \widetilde{Y}_{\varepsilon}, \alpha_{\varepsilon}\right)\right] & =\mathbb{E}\left[\mathbb{E}\left[h\left(\widetilde{Y}_{\tau_{\mathscr{D}}}\right) \circ \theta_{\varepsilon} \mid \mathscr{F}_{\varepsilon}\right]\right] \\
& =\mathbb{E}\left[h\left(\widetilde{Y}_{\tau_{\mathscr{D}}+\varepsilon}\right)\right] \leq \sup _{\tau \in \Lambda_{t, T}} \mathbb{E}\left[h\left(\widetilde{Y}_{\tau}\right)\right]=V(t, y, i),
\end{aligned}
$$


where the inequality follows from the fact that $\tau_{\mathscr{D}}(t+\varepsilon, y, i) \leq T-(t+\varepsilon)$. By Dynkin's formula and (A.2), we have

$$
\begin{aligned}
\mathbb{E}\left[V\left(t+\varepsilon, \tilde{Y}_{\varepsilon}, \alpha_{\varepsilon}\right)\right]-V(t, y, i) & =\mathbb{E}\left[e^{-r \varepsilon} V\left(t+\varepsilon, Y_{\varepsilon}, \alpha_{\varepsilon}\right)\right]-V(t, y, i) \\
& =\mathbb{E}\left[\int_{0}^{\varepsilon} e^{-r v}\left(V_{t}+\mathscr{A} V-r V\right)\left(t+v, Y_{v}^{y}, \alpha_{v}\right) \mathrm{d} v\right] \\
& \leq 0,
\end{aligned}
$$

where the last inequality follows from (A.7). Dividing the above inequality by $\varepsilon$, letting $\varepsilon \longrightarrow 0$, and using the mean value theorem, we derive

$$
\left(V_{t}+\mathscr{A} V-r V\right)(t, y, i) \leq 0,
$$

for any $(t, y, i) \in(0, T) \times(0,+\infty) \times \mathcal{S}$. Hence, the variational inequality (18) holds.

\section{B. Proof of Lemma 2}

Proof. For $0<y<G$, we immediately have $V(t, y, i) \geq(G-y)^{+}>0$. For $y>G$, let $\tau=(T-t) \wedge$ inf $\{t \geq 0: y M(t) \leq(G / 2)\}$ and observe that

$$
V(t, y, i) \geq \mathbb{E}\left[e^{-r \tau}(G-y M(\tau))^{+}\right] \geq \frac{G}{2} \mathbb{E}\left[e^{-r \tau}\right]>0 .
$$

This means $V$ is positive.

For $0 \leq t_{1}<t_{2} \leq T$, let $\tau$ be $\varepsilon$-optimal stopping time for $V\left(t_{2}, y, i\right)$. As $0 \leq \tau \leq T-t_{2}<T-t_{1}$ and $\tau$ is suboptimal for $V\left(t_{1}, y, i\right)$

$$
\begin{aligned}
V\left(t_{1}, y, i\right) & \geq \mathbb{E}^{i, y}\left[e^{-r \tau} h\left(Y_{\tau}\right)\right] \\
& \geq V\left(t_{2}, y, i\right)-\varepsilon
\end{aligned}
$$

Letting $\varepsilon \longrightarrow 0$, this implies $V$ is decreasing in $t$. It is easy to see that

$$
V(t, y, i)=\sup _{0 \leq \tau \leq T-t} \mathbb{E}^{i, y}\left[e^{-r \tau} \max \left(G-y M_{\tau}, 0\right)\right] .
$$

It follows that $\mathrm{V}$ is decreasing in $y$.

For any $y_{1}, y_{2}>0, \quad \lambda \in[0,1], \quad$ by setting $y^{*}=\lambda y_{1}+(1-\lambda) y_{2}$, we obtain that

$$
\begin{aligned}
V\left(t, y^{*}, i\right) & =\sup _{0 \leq \tau \leq T-t} \mathbb{E}\left[e^{-r \tau} h\left(Y_{\tau}^{y^{*}}\right)\right]=\sup _{0 \leq \tau \leq T-t} \mathbb{E}\left[e^{-r \tau} h\left(\lambda Y_{\tau}^{y_{1}}+(1-\lambda) Y_{\tau}^{y_{2}}\right)\right] \\
& \leq \lambda \sup _{0 \leq \tau \leq T-t} \mathbb{E}\left[e^{-r \tau} h\left(Y_{\tau}^{y_{1}}\right)\right]+(1-\lambda) \sup _{0 \leq \tau \leq T-t} \mathbb{E}\left[e^{-r \tau} h\left(Y_{\tau}^{y_{2}}\right)\right] \\
& =\lambda V\left(t, y_{1}, i\right)+(1-\lambda) V\left(t, y_{2}, i\right),
\end{aligned}
$$

which is the convexity of $V$ in $y$.

\section{Data Availability}

No data were used in this paper.

\section{Conflicts of Interest}

The authors declare that they have no conflicts of interest.

\section{Acknowledgments}

This work was supported by the Foundation of Yibin University, China (Grant no. 2019QD07).

\section{References}

[1] C. Bernard, A. MacKay, and M. Muehlbeyer, "Optimal surrender policy for variable annuity guarantees," Insurance: Mathematics and Economics, vol. 55, pp. 116-128, 2014.

[2] K. Ignatieva, A. Song, and J. Ziveyi, "Pricing and hedging of guaranteed minimum benefits under regime-switching and stochastic mortality," Insurance: Mathematics and Economics, vol. 70, pp. 286-300, 2016.

[3] D. Bauer, A. Kling, and J. Russ, "A universal pricing framework for guaranteed minimum benefits in variable annuities," ASTIN Bulletin, vol. 38, no. 2, pp. 621-651, 2008.

[4] Y. Shen, M. Sherris, and J. Ziveyi, "Valuation of guaranteed minimum maturity benefits in variable annuities with surrender options," Insurance: Mathematics and Economics, vol. 69, pp. 127-137, 2016.

[5] A. R. Bacinello, "Pricing guaranteed life insurance participating policies with annual premiums and surrender option," North American Actuarial Journal, vol. 7, pp. 1-17, 2013.

[6] J. Alonso-Garcia, M. Sherris, S. Thirurajah, and J. Ziveyi, "Taxation and policyholder behavior: the case of guaranteed minimum accumulation benefits," SSRN Electronic Journal, 2020.

[7] B. Kang and J. Ziveyi, "Optimal surrender of guaranteed minimum maturity benefits under stochastic volatility and interest rates," Insurance: Mathematics and Economics, vol. 79, pp. 43-56, 2018.

[8] J. Jeon and M. Kwak, "Optimal surrender strategies and valuations of path-dependent guarantees in variable 
annuities," Insurance: Mathematics and Economics, vol. 83, pp. 93-109, 2018.

[9] J. Jeon and G. Kim, "Efficient valuation of a variable annuity contract with a surrender option," Japan Journal of Industrial and Applied Mathematics, vol. 37, no. 1, pp. 249-262, 2020.

[10] A. MacKay and A. Ocejo, "Portfolio optimization with a guaranteed minimum maturity benefit and risk-adjusted fees," SSRN Electronic Journal, 2019.

[11] J. Buffington and R. J. Elliott, "American options with regime switching," International Journal of Theoretical and Applied Finance, vol. 5, no. 5, pp. 497-514, 2002.

[12] S. D. Jacka and A. Ocejo, "On the regularity of American options with regime-switching uncertainty," Stochastic Processes and Their Applications, vol. 128, no. 3, pp. 803-818, 2018.

[13] S. Boyarchenkp and S. Levendorski, "American options in regime-switching models," SIAM Journal on Control and Optimization, vol. 48, pp. 1353-1376, 2009.

[14] T. K. Siu, "Fair valuation of participating policies with surrender options and regime switching," Insurance: Mathematics and Economics, vol. 37, no. 3, pp. 533-552, 2005.

[15] Z. Yang, "A system of variational inequalities arising from finite expiry Russian option with two regimes," Mathematical Methods in the Applied Sciences, vol. 32, no. 13, pp. 1681-1703, 2009.

[16] F. L. Yuen and H. Yang, "Option pricing with regime switching by trinomial tree method," Journal of Computational and Applied Mathematics, vol. 233, no. 8, pp. 18211833, 2010.

[17] S.-P. Zhu, A. Badran, and X. Lu, "A new exact solution for pricing European options in a two-state regime-switching economy," Computers \& Mathematics with Applications, vol. 64, no. 8, pp. 2744-2755, 2012.

[18] G. Peskir and A. Shiryaev, Optimal Stopping and FreeBoundary Problems, Birkhauser, Basel, Switzerland, 2006.

[19] X. Mao and C. Yuan, Stochastic Differential Equations with Markovian Switching, Imperial College Press, London, UK, 2006.

[20] G. M. Lieberman, Second Order Parabolic Differential Equations, World Scientific, New Jersey, NJ, USA, 1996. 